TITLE:

\title{
Simulating two-dimensional infrared-Raman and Raman spectroscopies for intermolecular and intramolecular modes of liquid water
}

\section{$\operatorname{AUTHOR}(S):$}

Ito, Hironobu; Tanimura, Yoshitaka

\section{CITATION:}

Ito, Hironobu ... [et al]. Simulating two-dimensional infrared-Raman and Raman

spectroscopies for intermolecular and intramolecular modes of liquid water. The Journal of Chemical Physics 2016, 144(7): 074201.

\section{ISSUE DATE:}

2016-02-21

URL:

http://hdl.handle.net/2433/218326

\section{RIGHT:}

(C) 2016 AlP Publishing. This article may be downloaded for personal use only. Any other use requires prior permission of the author and AIP Publishing. The following article appeared in [J. Chem. Phys. 144, 074201 (2016); doi:

10.1063/1.49418429 and may be found at http://aip.scitation.org/doi/10.1063/1.4941842. 


\title{
Simulating two-dimensional infrared-Raman and Raman spectroscopies for intermolecular and intramolecular modes of liquid water
}

\author{
Hironobu Ito $^{\text {a) }}$ and Yoshitaka Tanimura ${ }^{\text {b) }}$ \\ Department of Chemistry, Graduate School of Science, Kyoto University, Sakyoku, Kyoto 606-8502, Japan
}

(Received 11 December 2015; accepted 1 February 2016; published online 18 February 2016)

\begin{abstract}
Full classical molecular dynamics (MD) simulations of two-dimensional (2D) infrared-Raman and 2D Raman spectroscopies of liquid water were carried out to elucidate a mode-mode coupling mechanism using a polarizable water model for intermolecular and intramolecular vibrational spectroscopy (POLI2VS). This model is capable of describing both infrared and Raman spectra. Second-order response functions, which consist of one molecular polarizability and two molecular dipole moments for 2D IR-Raman and three molecular polarizabilities for 2D Raman spectroscopies, were calculated using an equilibrium-non-equilibrium hybrid MD approach. The obtained signals were analyzed using a multi-mode Brownian oscillator (BO) model with nonlinear system-bath interactions representing the intramolecular $\mathrm{OH}$ stretching, intramolecular $\mathrm{HOH}$ bending, hydrogen bonded (HB)-intermolecular librational motion and HB-intermolecular vibrational (translational) motion of liquid water. This model was applied through use of hierarchal Fokker-Planck equations. The qualitative features of the peak profiles in the 2D spectra obtained from the MD simulations are accurately reproduced with the BO model. This indicates that this model captures the essential features of the intermolecular and intramolecular motion. We elucidate the mechanisms governing the 2D signal profiles involving anharmonic mode-mode coupling, the nonlinearities of the polarizability and dipole moment, and the vibrational dephasing processes of liquid water even in the case that the 2D spectral peaks obtained from the MD simulation overlap or are unclear. The mode coupling peaks caused by electrical anharmonic coupling (EAHC) and mechanical anharmonic coupling (MAHC) are observed in all of the 2D spectra. We find that the strength of the MAHC between the $\mathrm{OH}$-stretching and HB-intermolecular vibrational modes is comparable to that between the $\mathrm{OH}$-stretching and $\mathrm{HOH}$ bending modes. Moreover, we find that this $\mathrm{OH}$-stretching and $\mathrm{HB}$-intermolecular vibrational coupling should be observed as off-diagonal cross peaks in the 2D spectra. (C) 2016 AIP Publishing LLC. [http://dx.doi.org/10.1063/1.4941842]
\end{abstract}

\section{INTRODUCTION}

Intramolecular vibrational modes play essential roles in many chemical and biological processes because they promote bond forming and bond breaking processes in solute molecules in solvation. To realize irreversible chemical reaction processes, in which energy is not conserved, interplay between the interactions among intramolecular modes and intermolecular modes is essential. ${ }^{1-3}$ In numerical calculations employing classical molecular dynamics (MD) simulations, two-dimensional (2D) infrared (IR), and 2D heterodyne-detected vibrational sum frequency generation (HD-VSFG), and 2D IR surface spectra have been applied to study high-frequency intramolecular motion in bulk water, ${ }^{4-9}$ the vapor/water interface, ${ }^{9}$ and $\mathrm{CO}$ molecule on the $\mathrm{Cu}(100)$ surface, ${ }^{10}$ while 2D Raman ${ }^{11-18}$ and $2 \mathrm{D} \mathrm{THz}-$ $\operatorname{Raman}^{17-23}$ signals have been applied to detect low-frequency intermolecular vibrational modes. Theoretical studies of 2D spectroscopic approaches for intermolecular modes have demonstrated a capability to elucidate the difference

\footnotetext{
a)Electronic mail: h.ito@kuchem.kyoto-u.ac.jp

b)Electronic mail: tanimura@ @uchem.kyoto-u.ac.jp
}

between homogeneous and inhomogeneous broadening, ${ }^{24-27}$ the anharmonicity of the potential, ${ }^{28-30}$ and the modemode coupling mechanism. ${ }^{31-35}$ Also, interactions among the intermolecular modes have been investigated with $2 \mathrm{D}$ Raman and 2D THz-Raman spectroscopy, while those among the intramolecular modes have been investigated with $2 \mathrm{D}$ IR spectroscopy. The vibrational relaxation processes of $\mathrm{OH}$ stretching and $\mathrm{HOH}$ bending in liquid water has been investigated experimentally ${ }^{36-44}$ and theoretically ${ }^{2-9}$ by means of 2D IR. The results of these experiments indicate the importance of the coupling between the intramolecular and intermolecular modes. However, direct measurement of the interactions between these modes are difficult to carry out, because no practical light source that can cover both intermolecular and intramolecular modes has yet been developed.

In this paper, we theoretically examine the possibility of measuring interactions among intermolecular and intramolecular mode by means of 2D IR-Raman spectroscopy, in addition to 2D Raman spectroscopy, as an extension of 2D THz-Raman spectroscopy. ${ }^{20,45}$ We demonstrate this possibility specifically for liquid water using a polarizable water model for intermolecular and intramolecular vibrational spectroscopy 
$(\mathrm{POLI} 2 \mathrm{VS})^{46}$ that possesses the essential capability of simulating both infrared and Raman spectra. Both 2D Raman and 2D IR-Raman signals were calculated using the secondorder response functions expressed in terms of the threebody correlation functions of the single polarizability and two dipole moments in the 2D IR-Raman case and the three polarizabilities in the 2D Raman case. We calculated these signals using an equilibrium-nonequilibrium hybrid MD simulation algorithm. ${ }^{16}$ The calculated signals plotted in the frequency domain were analyzed using a Brownian oscillator (BO) model with linear-linear (LL) and square-linear (SL) system-bath interactions. ${ }^{24-35,47-51}$ To treat a non-perturbative, non-Markovian, nonlinear system-bath interaction, which is necessary to describe the effects of homogeneous and inhomogeneous broadening in a unified manner, we employed the hierarchal equations of motion (HEOM) approach. ${ }^{19,48-60}$

This paper is organized as follows. In Sec. II, we introduce the LL+SL BO model and the hierarchal equations of motion formalism for a multimode system. We then present the theoretical framework for analyzing 2D IR-Raman and 2D Raman spectra in the frequency domain on the basis of single-mode and two-mode models. In Sec. III, we describe the computational details of the full classical MD simulation of liquid water in the cases of 2D IR-Raman spectroscopy and 2D Raman spectroscopy. The MD and fitted results obtained from the LL+SL BO model are presented and analyzed in Sec. IV. Section V is devoted to concluding remarks.

\section{BROWNIAN ANALYSIS OF THE SECOND-ORDER RESPONSE FUNCTION}

\section{A. Multi-mode Brownian oscillator model with nonlinear system-bath coupling}

In 2D THz-Raman, 2D IR-Raman and 2D Raman spectroscopy methods, defined by second-order response functions expressed in terms of the three-body correlation functions of optical observables, the non-diagonal spectral peaks in the frequency domain signals need not be the modemode coupling peaks, because of overtone and population relaxation processes, ${ }^{31-35}$ unlike in the 2D IR case, defined by third-order response functions expressed in terms of the fourbody correlation functions of the dipole moment. ${ }^{1-3}$ Moreover, because the profiles of the peaks obtained in 2D spectroscopy studies employing MD approaches must be constructed from complex motion in a complicated manner, the analysis of the signal profile is not straightforward. It has been shown that model-based studies of the 2D profiles of signals are helpful for identifying the underlying physical mechanisms, because it is necessary to capture the essential features of the molecular vibrational motion in order to reproduce the complex 2D profile from a simple model. ${ }^{40,51}$ Such analyses of the BO model with nonlinear system-bath interactions have been carried out in the 2D Raman ${ }^{57-60}$ and 2D THz-Raman ${ }^{18,19}$ cases for intermolecular modes and in the 2D IR case for intramolecular modes. ${ }^{48-51}$ It has been shown that the analysis of 2D Raman signals based on the BO model is consistent with the normal mode analysis based on MD simulations. ${ }^{61}$ Here, we employ this model in the multi-mode case for calculations of the $2 \mathrm{D}$ spectra in the frequency domain in order to capture the characteristic features of the 2D signals and elucidate the role of the mode-mode coupling mechanism from the MD results.

We consider a model that consists of several primary oscillator modes independently coupled to the other optically inactive modes, which are regarded as a bath system. The bath system is represented by an ensemble of harmonic oscillators. The vibrational frequency of the primary mode may change in time or it can be made to be inhomogeneously distributed by introducing a nonlinear system-bath coupling. The total Hamiltonian is expressed as

$$
H=\sum_{s}\left(H_{\mathrm{S}}^{(s)}+H_{\mathrm{B}}^{(s)}+H_{\mathrm{I}}^{(s)}\right)+\sum_{s, s^{\prime}>s} U_{s, s^{\prime}}\left(q_{s}, q_{s^{\prime}}\right),
$$

where

$$
H_{\mathrm{S}}^{(s)}=\frac{p_{s}^{2}}{2 m_{s}}+U_{s}\left(q_{s}\right)
$$

is the Hamiltonian for the $s$ th mode with mass $m_{s}$, coordinate $q_{s}$, momentum $p_{s}$ and potential $U_{s}\left(q_{s}\right), U_{s s^{\prime}}\left(q_{s}, q_{s^{\prime}}\right)$ is the interaction between the modes $s$ and $s^{\prime}$,

$$
H_{\mathrm{B}}^{(s)}=\sum_{j_{s}}\left(\frac{p_{j_{s}}^{2}}{2 m_{j_{s}}}+\frac{m_{j_{s}} \omega_{j_{s}}^{2} x_{j_{s}}^{2}}{2}\right)+\sum_{j_{s}}\left(\frac{\alpha_{j_{s}}^{2} V_{s}^{2}\left(q_{s}\right)}{2 m_{j_{s}} \omega_{j_{s}}^{2}}\right)
$$

is the bath Hamiltonian for the $s$ th mode with the momentum, coordinate, mass, and frequency of the $j_{s}$ th bath oscillator given by $p_{j_{s}}, x_{j_{s}}, m_{j_{s}}$ and $\omega_{j_{s}}$, respectively, and

$$
H_{\mathrm{I}}^{(s)}=-V_{s}\left(q_{s}\right) \sum_{j_{s}} \alpha_{j_{s}} x_{j_{s}}
$$

is the system-bath interaction, which consists of linearlinear (LL) and square-linear (SL) system-bath interactions, $V_{s}\left(q_{s}\right) \equiv V_{\mathrm{LL}}^{(s)} q_{s}+V_{\mathrm{SL}}^{(s)} q_{s}{ }^{2} / 2$, with coupling strengths $V_{\mathrm{LL}}^{(s)}, V_{\mathrm{SL}}^{(s)}$, and $\alpha_{j_{s}}{ }^{4}{ }^{4}$ This model has been used to derive predictions of a single-mode system for 2D Raman ${ }^{19,27,57-60}$ and 2D IR signals ${ }^{48,49}$ and a two-mode system for $2 \mathrm{D}$ IR signal. ${ }^{50,51}$ The last term of the bath Hamiltonian is a counter-term, which maintains the translational symmetry of the system in the case $U_{s}\left(q_{s}\right)=U_{s, s^{\prime}}\left(q_{s}, q_{s^{\prime}}\right)=0 .{ }^{57}$ Here, we apply this model to describe the simulated $2 \mathrm{D}$ signal of liquid water. In this case, the vibrational frequencies of the representative modes all differ sufficiently that we can assume that the fluctuations arising from the bath are uncorrelated. In addition, the 2D vibrational spectroscopy considered here is insensitive to correlations among fluctuations, because it does not include a population decay time, unlike third-order spectroscopy. ${ }^{44,50,51}$ For this reason, we assume that each mode is coupled to a separate bath.

For the system potential and mode-mode interaction, we consider

$$
U_{s}\left(q_{s}\right)=\frac{1}{2 !} m_{s} \omega_{s}^{2} q_{s}^{2}+\frac{1}{3 !} g_{s^{3}} q_{s}^{3}
$$

and

$$
U_{s s^{\prime}}\left(q_{s}, q_{s^{\prime}}\right)=\frac{1}{2}\left(g_{s^{2} s^{\prime}} q_{s}^{2} q_{s^{\prime}}+g_{s s^{\prime 2}} q_{s} q_{s^{\prime}}^{2}\right) .
$$

We assume that the anharmonicity of the vibrational motion is weak and that the system potential can be expressed 
as a function of $q_{s}$ and $q_{s^{\prime}}$ in the form of a Taylor expansion that includes only terms through third order. It should be noted that the fourth-order term in this expansion does not contribute to the second-order response functions. ${ }^{28,29,31}$ As shown in Ref. 51, while the LL interaction contributes mainly to energy relaxation, the SL systembath interaction leads to vibrational dephasing in the slow modulation case, due to the frequency fluctuation of system vibrations. $^{51,58-60}$ The sum of the bath coordinates $X_{S}$ $\equiv \sum_{j_{s}} \alpha_{j_{s}} x_{j_{s}}$ acts as a collective coordinate that modulates the mode $s .{ }^{57}$ We introduce the spectral distribution function $J_{s}(\omega)$ $\equiv \sum_{j_{s}} \alpha_{j_{s}}^{2} \hbar \delta\left(\omega-\omega_{j_{s}}\right) / 2 m_{j_{s}} \omega_{j_{s}}$, which characterizes the bath and system-bath coupling. We assume that $J_{s}(\omega)$ has an Ohmic form with a Lorentzian cutoff: ${ }^{57}$

$$
J_{s}(\omega)=\frac{\hbar m_{s} \zeta_{s}}{2 \pi} \frac{\omega \gamma_{s}^{2}}{\gamma_{s}^{2}+\omega^{2}} .
$$

Here, $\zeta_{s}$ is the system-bath coupling strength, and $\gamma_{s}$ represents the width of the spectral distribution for the mode $s$.

In the present study, we consider each primary mode representing the collective modes of the $\mathrm{OH}$ stretching, $\mathrm{HOH}$ bending, HB-intermolecular librational and HBintermolecular vibrational (translational) motion of liquid water.

\section{B. Classical hierarchal Fokker-Planck equations for a multi-mode system}

To study a multi-mode Brownian system, we employ the reduced hierarchal equations of motion (HEOM) approach, which allows us to treat the effects of thermal activation, relaxation, the anharmonicity of the potential, the nonlinearities of the polarizability and dipole moment, vibrational dephasing, and homogeneous and inhomogeneous broadening in the 2D spectra within a unified framework. ${ }^{48-60}$ Although quantum mechanical effects are important to calculate the signals for high-frequency intramolecular modes ${ }^{49}$ here we employ a classical framework for the analysis of both intramolecular and intermolecular modes, because the MD results that allow comparison with the model calculations are all classical. For the LL+SL BO model with the two modes $\alpha$ and $\beta$, described by Eqs. (1)-(4), the HEOM for the classical distribution function, $W(\boldsymbol{p}, \boldsymbol{q} ; t)$ $\equiv W\left(p_{\alpha}, p_{\beta}, q_{\alpha}, q_{\beta} ; t\right)$, are obtained through the extension of a single-mode case as ${ }^{19,57}$

$$
\begin{aligned}
\frac{\partial W^{\left(n_{1}, n_{2}\right)}(\boldsymbol{p}, \boldsymbol{q} ; t)}{\partial t}= & -\left(\hat{L}_{\alpha+\beta}+n_{1} \gamma_{\alpha}+n_{2} \gamma_{\beta}\right) W^{\left(n_{1}, n_{2}\right)}(\boldsymbol{p}, \boldsymbol{q} ; t) \\
& -n_{1} \gamma_{\alpha} \hat{\Theta}_{\alpha} W^{\left(n_{1}-1, n_{2}\right)}(\boldsymbol{p}, \boldsymbol{q} ; t) \\
& -n_{2} \gamma_{\beta} \hat{\Theta}_{\beta} W^{\left(n_{1}, n_{2}-1\right)}(\boldsymbol{p}, \boldsymbol{q} ; t) \\
& -\hat{\Phi}_{\alpha} W^{\left(n_{1}+1, n_{2}\right)}(\boldsymbol{p}, \boldsymbol{q} ; t) \\
& -\hat{\Phi}_{\beta} W^{\left(n_{1}, n_{2}+1\right)}(\boldsymbol{p}, \boldsymbol{q} ; t),
\end{aligned}
$$

where $n_{1}$ and $n_{2}$ are the depth of the hierarchy elements for the modes $\alpha$ and $\beta$, respectively. In the HEOM approach, only the first element, $W^{(0,0)}(\boldsymbol{p}, \boldsymbol{q} ; t)$, has physical meaning, while the other elements, $W^{\left(n_{1}, n_{2}\right)}(\boldsymbol{p}, \boldsymbol{q} ; t)$ with $n_{1}$, $n_{2}>0$, are introduced into the numerical calculations in order to properly treat the non-perturbative, non-Markovian system-bath interactions. The classical Liouvillian of the system with the Hamiltonian $H_{\alpha+\beta} \equiv H_{\mathrm{S}}^{(\alpha)}+H_{\mathrm{S}}^{(\beta)}+U_{\alpha \beta}\left(q_{\alpha}, q_{\beta}\right)$ is given by

$$
\hat{L}_{\alpha+\beta}=\sum_{s}^{\alpha, \beta}\left(\frac{\partial H_{\alpha+\beta}}{\partial p_{s}} \frac{\partial}{\partial q_{s}}-\frac{\partial H_{\alpha+\beta}}{\partial q_{s}} \frac{\partial}{\partial p_{s}}\right) .
$$

The operators $\hat{\Theta}_{s}$ and $\hat{\Phi}_{s}$ here describe the energy exchange between the system and the heat bath for the $s$ th mode. These are defined in terms of the inverse correlation time, $\gamma_{s}$, the coupling strength, $\zeta_{s}$, and the temperature, $T$, as

$$
\hat{\Phi}_{s} \equiv-\frac{\partial V^{(s)}\left(q_{s}\right)}{\partial q_{s}} \frac{\partial}{\partial p_{s}}
$$

and

$$
\hat{\Theta}_{s} \equiv-\zeta_{s} \frac{\partial V^{(s)}\left(q_{s}\right)}{\partial q_{s}}\left(p_{s}+m_{s} k_{B} T \frac{\partial}{\partial p_{s}}\right),
$$

where $k_{B}$ is the Boltzmann constant. The equations of motion are truncated by making the replacements $\quad \hat{\Phi}_{\alpha} W^{\left(n_{1}+1, n_{2}\right)}(\boldsymbol{p}, \boldsymbol{q} ; t) \rightarrow-\hat{\Phi}_{\alpha} \hat{\Theta}_{\alpha} W^{\left(n_{1}, n_{2}\right)}(\boldsymbol{p}, \boldsymbol{q} ; t) \quad$ and $\hat{\Phi}_{\beta} W^{\left(n_{1}, n_{2}+1\right)}(\boldsymbol{p}, \boldsymbol{q} ; t) \rightarrow-\hat{\Phi}_{\beta} \hat{\Theta}_{\beta} W^{\left(n_{1}, n_{2}\right)}(\boldsymbol{p}, \boldsymbol{q} ; t)$ in Eq. (8) for sufficiently large $n_{1}+n_{2}=N .^{54-56}$ In principle, the HEOM provide an asymptotic approach that allows us to calculate various physical quantities with any desired accuracy by adjusting the number of hierarchal elements, determined by $N$; the error introduced by the truncation can be made negligibly small by choosing $N$ to be sufficiently large. The computational details for the hierarchal Fokker-Planck equations are described in Appendix A.

\section{Calculating 1D and 2D signals from response functions}

The optical observables in 1D and 2D spectroscopy studies are defined respectively as the first- and second-order response functions. ${ }^{24}$ In the classical case, they are expressed as ${ }^{17-19}$

$$
R\left(t_{1}\right)=-\left\langle\left\{A\left(t_{1}\right), B(0)\right\}_{\mathrm{PB}}\right\rangle
$$

and

$$
R\left(t_{2}, t_{1}\right)=\left\langle\left\{\left\{A\left(t_{2}+t_{1}\right), B\left(t_{1}\right)\right\}_{\mathrm{PB}}, C(0)\right\}_{\mathrm{PB}}\right\rangle,
$$

where $A, B$, and $C$ are the total dipole moment $(\boldsymbol{\mu})$ or the total polarizability $(\boldsymbol{\Pi})$ of the molecular system, and $\{,\}_{\mathrm{PB}}$ is the Poisson bracket defined by

$$
\{A, B\}_{\mathrm{PB}} \equiv \sum_{s}\left(\frac{\partial A}{\partial q_{s}} \frac{\partial B}{\partial p_{s}}-\frac{\partial A}{\partial p_{s}} \frac{\partial B}{\partial q_{s}}\right) .
$$

2D IR-Raman spectroscopy is described in terms of threebody correlation functions, as in the cases of 2D Raman and 2D THz-Raman spectroscopy. 2D IR-Raman spectroscopy utilizes two IR (or THz) processes and one Raman process, and there are three different measurements, specifically, those with the Raman-IR-IR (RII), IR-Raman-IR (IRI), and IR-IRRaman (IIR) configurations depicted in Fig. 1. In the present study, we also calculated the 2D Raman spectrum utilizing three Raman processes, because the recently developed single-beam spectrally controlled 2D Raman spectroscopy 

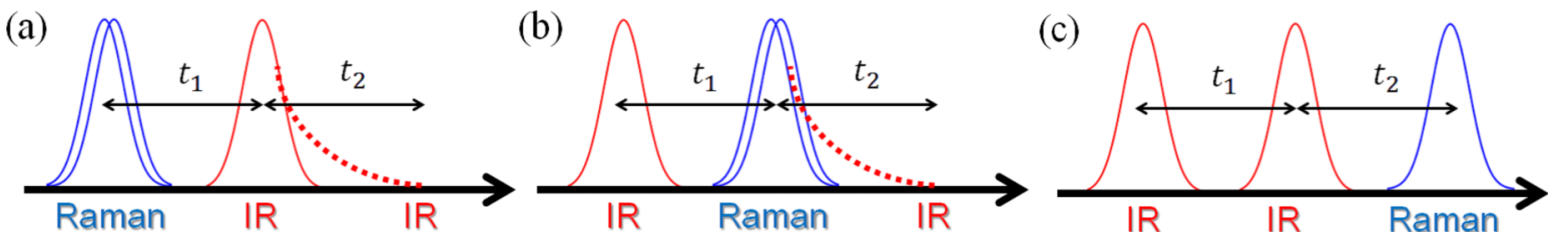

FIG. 1. Pulse sequences in the (a) Raman-IR-IR, (b) IR-Raman-IR, and (c) IR-IR-Raman configurations for 2D IR-Raman spectroscopy. The blue curves represent Raman interactions, whereas the red ones represent IR (or THz) interactions.

method makes it possible to obtain 2D spectra by suppressing cascading effects. ${ }^{66} \mathrm{We}$ can calculate these signals by setting, for example, $A=\mu(\boldsymbol{q}), B=\mu(\boldsymbol{q})$, and $C=\Pi(\boldsymbol{q})$ in Eq. (13) for the RII case.

To apply the HEOM formalism, we express the response functions in terms of Wigner distribution functions as ${ }^{30,57}$

$$
R\left(t_{1}\right)=\iint d \boldsymbol{p} d \boldsymbol{q}\left\{-A(\boldsymbol{q}) \mathcal{G}\left(t_{1}\right) B^{\times}(\boldsymbol{q}) W^{\mathrm{eq}}(\boldsymbol{p}, \boldsymbol{q})\right\}
$$

and

$$
R\left(t_{2}, t_{1}\right)=\iint d \boldsymbol{p} d \boldsymbol{q} A(\boldsymbol{q}) \mathcal{G}\left(t_{2}\right) B^{\times}(\boldsymbol{q}) \mathcal{G}\left(t_{1}\right) C^{\times}(\boldsymbol{q}) W^{\mathrm{eq}}(\boldsymbol{p}, \boldsymbol{q}),
$$

where we have employed the hyperoperator ${ }^{x}$ defined as $C^{\times}(\boldsymbol{q}) W(\boldsymbol{p}, \boldsymbol{q}) \equiv\{C(\boldsymbol{q}), W(\boldsymbol{p}, \boldsymbol{q})\}_{\mathrm{PB}}$, and $\mathcal{G}(t)$ is the Green's function of the system Hamiltonian without a laser pulse interaction. Then, the 1D spectra are obtained from the above as

$$
I(\omega)=\omega \mathfrak{J} \int_{0}^{\infty} d t e^{i \omega t_{1}} R\left(t_{1}\right),
$$

where the pre-factor $\omega$ for the 1D spectrum is a harmonic quantum correction factor that must be included with the classical calculations. ${ }^{62}$ By contrast, the $2 \mathrm{D}$ spectra are estimated as

$I\left(\omega_{2}, \omega_{1}\right)=\iint_{0}^{\infty} R\left(t_{2}, t_{1}\right) \sin \left(\omega_{1} t_{1}\right) \sin \left(\omega_{2} t_{2}\right) d t_{1} d t_{2}$.

In the low-frequency range, this $2 \mathrm{D}$ sine-Fourier representation is more intuitive than the real part of the 2D Fourier representation. ${ }^{20}$

Although the characteristic features of 2D Raman signals in Fourier space have been explored on the basis of analytic solutions of the LL Brownian oscillator model, ${ }^{31-35}$ the extension of these studies to 2D IR-Raman and 2D THz-Raman spectroscopy on the basis of the LL+SL BO model have not yet been explored, particularly for multimode systems. Using the HEOM approach, here we investigate the roles of anharmonic mode-mode coupling with inhomogeneous broadening for such $2 \mathrm{D}$ spectra described by this model.

As shown in the cases of 2D Raman and 2D THz-Raman spectroscopy, nonlinear polarizability plays an essential role in $2 \mathrm{D}$ spectroscopy results described by the second-order response function. ${ }^{21,24}$ In the 2D IR-Raman case, we find that the nonlinear dipole elements also play a significant role, because the induced contributions of the dipole moment for the $\mathrm{OH}$ stretching and HB-intermolecular vibrational modes are very large in comparison with those for the other modes, as illustrated in Appendix C. Thus, we assume

$$
\mu(\boldsymbol{q})=\sum_{s} \mu_{s} q_{s}+\frac{1}{2 !} \sum_{s, s^{\prime}} \mu_{s s^{\prime}} q_{s} q_{s^{\prime}}
$$

and

$$
\Pi(\boldsymbol{q})=\sum_{s} \Pi_{s} q_{s}+\frac{1}{2 !} \sum_{s, s^{\prime}} \Pi_{s s^{\prime}} q_{s} q_{s^{\prime}}
$$

where $\mu_{s}$ and $\mu_{s s^{\prime}}$ are the linear and nonlinear elements of the dipole moment, and $\Pi_{s}$, and $\Pi_{s s^{\prime}}$ are those of the polarizability, respectively. As in the case of 2D Raman spectroscopy, the 2D signal defined in terms of the second-order response function, given in Eq. (16), will vanish when the system is harmonic and the optical property is linear, because in that case, there is an odd number of Gaussian integrals involved in the response function: $\operatorname{tr}\left[\boldsymbol{q}\left(t_{2}+t_{1}\right) \boldsymbol{q}\left(t_{1}\right) \boldsymbol{q}(0) \exp \left(-H_{S} / k_{B} T\right)\right]^{28-30}$ To illustrate the characteristic features of the $2 \mathrm{D}$ profile in the frequency domain spectrum, here we study the anharmonic (AH) contribution and nonlinear contribution (NL) separately. For this purpose, we consider the following three elements:

$$
\begin{aligned}
R_{\mathrm{AH}}\left(t_{2}, t_{1}\right)= & \sum_{s, s^{\prime}, s^{\prime \prime}} \mu_{s} \mu_{s^{\prime}} \Pi_{s^{\prime \prime}} \\
& \times\left\langle\left\{\left\{q_{s}\left(t_{2}+t_{1}\right), q_{s^{\prime}}\left(t_{1}\right)\right\}_{\mathrm{PB}}, q_{s^{\prime \prime}}(0)\right\}_{\mathrm{PB}}\right\rangle, \\
R_{\mathrm{NL} 2}\left(t_{2}, t_{1}\right)= & \frac{1}{2} \sum_{s, s^{\prime}, s^{\prime \prime}, s^{\prime \prime \prime}} \mu_{s} \Pi_{s^{\prime} s^{\prime \prime}} \mu_{s^{\prime \prime \prime}} \\
& \times\left\langle\left\{\left\{q_{s}\left(t_{2}+t_{1}\right), q_{s^{\prime}}\left(t_{1}\right) q_{s^{\prime \prime}}\left(t_{1}\right)\right\}_{\mathrm{PB}}, q_{s^{\prime \prime \prime}}(0)\right\}_{\mathrm{PB}}\right\rangle,
\end{aligned}
$$

and

$$
\begin{aligned}
R_{\mathrm{NL} 3}\left(t_{2}, t_{1}\right)= & \frac{1}{2} \sum_{s, s^{\prime}, s^{\prime \prime}, s^{\prime \prime \prime}} \Pi_{s s^{\prime}} \mu_{s^{\prime \prime}} \mu_{s^{\prime \prime \prime}} \\
& \times\left\langle\left\{\left\{q_{s}\left(t_{2}+t_{1}\right) q_{s^{\prime}}\left(t_{2}+t_{1}\right), q_{s^{\prime \prime}}\left(t_{1}\right)\right\}_{\mathrm{PB}}, q_{s^{\prime \prime \prime}}(0)\right\}_{\mathrm{PB}}\right\rangle .
\end{aligned}
$$

As has been demonstrated in the case of single-mode 2D THz-Raman spectroscopy, these elements correspond to the RII, IRI, and IIR signals, respectively, for $\mu_{s s^{\prime}}=0$. It should be noted that the NL term that corresponds to RII,

$$
\begin{aligned}
R_{\mathrm{NL} 1}\left(t_{2}, t_{1}\right)= & \frac{1}{2} \sum_{s, s^{\prime}, s^{\prime \prime}, s^{\prime \prime \prime}} \mu_{s} \mu_{s^{\prime}} \Pi_{s^{\prime \prime} s^{\prime \prime \prime}} \\
& \times\left\langle\left\{\left\{q_{s}\left(t_{2}+t_{1}\right), q_{s^{\prime}}\left(t_{1}\right)\right\}_{\mathrm{PB}}, q_{s^{\prime \prime}}(0) q_{s^{\prime \prime \prime}}(0)\right\}_{\mathrm{PB}}\right\rangle
\end{aligned}
$$

is weak or even vanishing. This point can be demonstrated using diagram theory ${ }^{31-35}$ and model calculations. ${ }^{18,19}$ 
TABLE I. Parameter values for the anharmonicity and optical properties in the single-mode SL BO model.

\begin{tabular}{llllc}
\hline \hline Contribution & $\bar{g}_{\alpha^{3}}$ & $\bar{\mu}_{\alpha}$ & $\bar{\Pi}_{\alpha}$ & $\bar{\Pi}_{\alpha \alpha}$ \\
\hline AH & 0.21 & 1.0 & 1.0 & $\ldots$ \\
NL2 & 0 & 1.0 & $\ldots$ & 0.10 \\
NL3 & 0 & 1.0 & $\ldots$ & 0.10 \\
\hline \hline
\end{tabular}

\section{Characteristic features of the $2 D$ spectrum in the single-mode case}

First, we elucidate the characteristic features of the AH and NL contributions in the single-mode case described by the SL BO model. In the numerical calculations, we employed dimensionless coordinates, system-bath coupling strengths, LL coupling strengths, and SL coupling strengths defined by $\bar{q}_{s} \equiv \sqrt{m_{s} \omega_{0} / \hbar} q_{s}, \bar{\zeta}_{s} \equiv \zeta_{s} / \omega_{0}, \bar{V}_{L L}^{(s)} \equiv V_{L L}^{(s)}$, and $\bar{V}_{S L}^{(s)} \equiv \sqrt{\hbar / m_{s} \omega_{0}} V_{S L}^{(s)}$. The potential is then expressed as

$$
U_{s}\left(\bar{q}_{s}\right)=\frac{1}{2} \hbar \omega_{0}\left(\frac{\omega_{s}}{\omega_{0}}\right)^{2} \bar{q}_{s}^{2}+\frac{1}{3 !} \hbar \omega_{0} \bar{g}_{s} \bar{q}_{s}^{3},
$$

where $\bar{g}_{s^{3}}$ is the cubic anharmonicity of the potential. We also employ the following normalized optical variables $\bar{\mu}_{s} \equiv \sqrt{\hbar / m_{s} \omega_{0}} \mu_{s}, \quad \bar{\mu}_{s^{2}} \equiv \sqrt{\hbar^{2} / m_{s}^{2} \omega_{0}^{2}} \mu_{s^{2}}, \quad \bar{\Pi}_{s} \equiv \sqrt{\hbar / m_{s} \omega_{0}} \Pi_{s}$, and $\bar{\Pi}_{s^{2}} \equiv \sqrt{\hbar^{2} / m_{s}^{2} \omega_{0}^{2}} \Pi_{s^{2}}$. In this case, Eq. (8) was simplified by reducing the hierarchal elements for the second mode, $\beta$, described by $n_{2}{ }^{19}$ The system and heat bath parameter values were chosen as $\omega_{\alpha}=1.0, \bar{\zeta}_{\alpha}=0.50 \omega_{0}, \gamma_{\alpha}=0.50 \omega_{0}$, $\bar{V}_{L L}^{(\alpha)}=0$ and $\bar{V}_{S L}^{(\alpha)}=1.0$ with $\omega_{0}=1.0$ and $\hbar \omega_{0} / k_{B} T=1.0$. The other parameter values are presented in Table I.

In Fig. 2, we display the 2D spectra for the (i) $\mathrm{AH}$, (ii) NL2, and (iii) NL3 contributions calculated using Eqs. (21)-(23). The 2D spectrum of the AH contribution exhibits positive and negative peaks labeled by "A" along the diagonal direction. These arise from the interplay between the linear polarizability and the cubic anharmonicity. The NL2 contribution labeled by " $\mathbf{A}$ " arises from the nonlinear polarizability. The diagonal peak in the NL2 case is identified as an echo peak, which represents the rephrasing process. The AH and NL3 contributions also exhibit spectral peaks along the off-diagonal direction, labeled by "B" and "C'. It should be noted that the off-diagonal peaks in $2 \mathrm{D}$ spectroscopy spectra defined in terms of the second-order response function need not be the mode-mode coupling peaks, as in the case of 2D IR spectroscopy defined in terms of the third-order response function. ${ }^{31-35}$ The spectral peaks near $\left(\omega_{1}, \omega_{2}\right)=\left(\omega_{\alpha}, 2 \omega_{\alpha}\right)$ labeled by "B" correspond to the overtone peak in the $\omega_{2}$ direction. The broadened peaks near $\left(\omega_{1}, \omega_{2}\right)=\left(\omega_{\alpha}, 0\right)$ labeled by "C" arise from population relaxation; a signal that relaxes as $e^{-\gamma t_{2}}$ along the $t_{2}$ axis with the relaxation rate $\gamma$ appears as $\int_{0}^{\infty} e^{-\gamma t_{2}} \sin \left(\omega_{2} t_{2}\right) d t_{2}=\omega_{2} /\left(\gamma^{2}+\omega_{2}^{2}\right)$ in $2 \mathrm{D}$ spectrum. Although our simulation results are classical, these peak positions can be interpreted readily using the optical Liouville paths, as discussed in Ref. 19.

\section{Characteristic features of $2 D$ signals in the two-mode case}

Next, we consider the two-mode case. Here, the normalized potential for mode-mode interaction is expressed as

$U_{s s^{\prime}}\left(\bar{q}_{s}, \bar{q}_{s^{\prime}}\right)=\frac{1}{3 !} \hbar \omega_{0}\left(3 \bar{g}_{s^{2} s^{\prime}} \bar{q}_{s}^{2} \bar{q}_{s^{\prime}}+3 \bar{g}_{s s^{\prime 2}} \bar{q}_{s} \bar{q}_{s^{\prime}}^{2}\right)$,

where $\bar{g}_{s^{2} s^{\prime}}$ and $\bar{g}_{s s^{\prime 2}}$ represent the cubic anharmonic coupling of the potential. We employ the following nonlinear optical coupling variables: $\bar{\mu}_{s s^{\prime}} \equiv \sqrt{\hbar^{2} / m_{s} m_{s^{\prime}} \omega_{0}^{2}} \mu_{s s^{\prime}}$ and $\bar{\Pi}_{s s^{\prime}} \equiv \sqrt{\hbar^{2} / m_{s} m_{s^{\prime}} \omega_{0}^{2}} \Pi_{s s^{\prime}}$

We fixed the system and bath parameter values as $\gamma_{\alpha}=\gamma_{\beta}$ $=0.50 \omega_{0}, \bar{V}_{L L}^{(\alpha)}=\bar{V}_{L L}^{(\beta)}=0, \bar{V}_{S L}^{(\alpha)}=\bar{V}_{S L}^{(\beta)}=1.0, \bar{g}_{\alpha^{3}}=\bar{g}_{\beta^{3}}=0$, and $\bar{\Pi}_{\alpha \alpha}=\bar{\Pi}_{\beta \beta}=0$, with $\omega_{0}=1.0$ and $\hbar \omega_{0} / k_{B} T=1.0$. The other parameter values are presented in Table II. In the twomode case, the vibrational modes interact through electrical anharmonic coupling (EAHC) and/or mechanical anharmonic coupling (MAHC) ${ }^{31-35}$ In Fig. 3, we present the 2D spectra in the (i) AH1 and (ii) AH2 cases (arising from the MAHC) and the (iii) NL2 and (iv) NL3 cases (arising from the EAHC), calculated on the basis of the SL BO model. The spectra in (a) are those for the high-frequency modes, and the spectra in (b) are those for the low-frequency modes.

For the high-frequency modes, as in the single-mode case depicted in Fig. 2(i), the diagonal peak, overtone peak, and relaxation peak labeled by "A," "B," and "C" for the two modes $\alpha$ and $\beta$ are observed as the peaks labeled by "A," "B," and "C" in Figs. 3(a-i) and 3(a-ii),
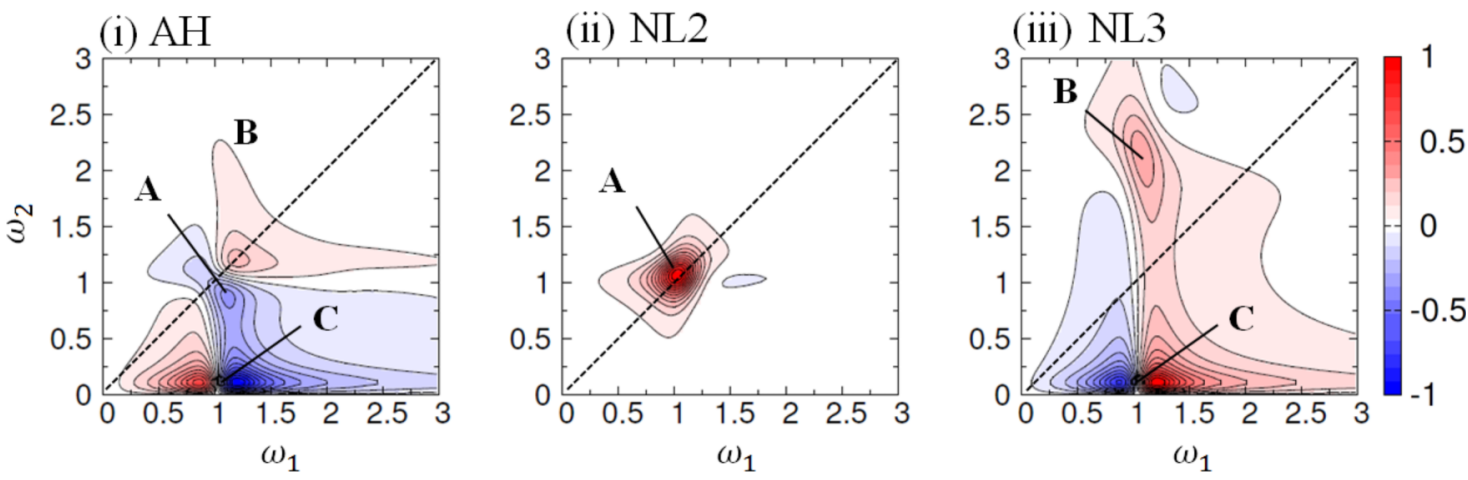

FIG. 2. 2D spectra of the single-mode system for the (i) AH, (ii) NL2, and (iii) NL3 contributions calculated using the one-mode SL BO model. The spectral intensities are normalized. 
TABLE II. Parameter values of the anharmonicity and optical properties for the two-mode SL BO model. We fixed the frequencies and bath coupling strengths of the modes $\alpha$ and $\beta$ as (a) $\omega_{\alpha}=4.0, \omega_{\beta}=1.0, \bar{\zeta}_{\alpha}=1.3 \omega_{0}$, $\bar{\zeta}_{\beta}=0.5 \omega_{0}$ and (b) $\omega_{\alpha}=1.0, \omega_{\beta}=0.20, \bar{\zeta}_{\alpha}=0.5 \omega_{0}, \bar{\zeta}_{\beta}=1.0 \times 10^{-2} \omega_{0}$, with $\omega_{0}=1.0$ and $\hbar \omega_{0} / k_{B} T=1.0$.

\begin{tabular}{|c|c|c|c|c|c|c|c|c|}
\hline Case (a) & $\bar{g}_{\alpha^{2} \beta}$ & $\bar{g}_{\alpha \beta^{2}}$ & $\bar{\mu}_{\alpha}$ & $\bar{\mu}$ & & $\overline{\mathrm{I}}_{\alpha}$ & $\bar{\Pi}_{\beta}$ & $\bar{\Pi}_{\alpha \beta}$ \\
\hline AH1 & -0.42 & 0 & 1.0 & 1. & & .0 & 1.0 & $\ldots$ \\
\hline $\mathrm{AH} 2$ & 0 & -0.11 & 1.0 & 1. & & .0 & 1.0 & $\ldots$ \\
\hline NL2 & 0 & 0 & 1.0 & 1. & & .. & $\ldots$ & 0.10 \\
\hline NL3 & 0 & 0 & 1.0 & 1. & & .. & $\ldots$ & 0.10 \\
\hline Case (b) & $\bar{g}_{\alpha^{2} \beta}$ & \multicolumn{2}{|c|}{$\bar{g}_{\alpha \beta^{2}}$} & $\bar{\mu}_{\alpha}$ & $\bar{\mu}_{\beta}$ & $\bar{\Pi}_{\alpha}$ & $\bar{\Pi}_{\beta}$ & $\bar{\Pi}_{\alpha \beta}$ \\
\hline AH1 & $-9.0 \times 10^{-3}$ & \multicolumn{2}{|l|}{0} & 1.0 & 1.0 & 1.0 & 1.0 & . \\
\hline $\mathrm{AH} 2$ & 0 & \multicolumn{2}{|c|}{$-1.8 \times 10^{-3}$} & 1.0 & 1.0 & 1.0 & 1.0 & $\ldots$ \\
\hline NL2 & 0 & \multicolumn{2}{|l|}{0} & 1.0 & 1.0 & $\ldots$ & $\ldots$ & 0.10 \\
\hline NL3 & 0 & \multicolumn{2}{|l|}{0} & 1.0 & 1.0 & $\ldots$ & $\ldots$ & 0.10 \\
\hline
\end{tabular}

respectively. In addition, in the AH1, AH2 an NL2 spectra in this case, these appear off-diagonal peaks near $\left(\omega_{1}, \omega_{2}\right)=\left(\omega_{\beta}, \omega_{\alpha}\right)$ and $\left(\omega_{1}, \omega_{2}\right)=\left(\omega_{\alpha}, \omega_{\beta}\right)$, labeled by "D" and "E." Also, for the high-frequency modes, there are peaks near the positions, $\left(\omega_{1}, \omega_{2}\right)=\left(\omega_{\beta}, \omega_{\alpha}+\omega_{\beta}\right)$ and $\left(\omega_{1}, \omega_{2}\right)=\left(\omega_{\alpha}, \omega_{\alpha}+\omega_{\beta}\right)$, labeled by "F" and " $\mathbf{F}$ "' and peaks near $\left(\omega_{1}, \omega_{2}\right)=\left(\omega_{\beta}, \omega_{\alpha}-\omega_{\beta}\right)$ and $\left(\omega_{1}, \omega_{2}\right)=\left(\omega_{\alpha}, \omega_{\alpha}-\omega_{\beta}\right)$, labeled by "G" and "G" in the AH1, AH2, and NL3 spectra. The peaks labeled by "D," "E," "F," "G," "F'," and "G", in Figs. 3(a-iii) and 3(a-iv) arise from the EAHC. These peak positions can be interpreted easily using the optical Liouville paths depicted in Fig. 4. The peaks in Figs. 3(a-i) and 3(a-ii) arise from the MAHC. Despite this fact, however, the peaks labeled by "A," "B," "C," "D," "E," "F," "G," "F'" and "G" appear at the same positions as those in the NL2 and NL3 spectra in the single-mode and two-mode cases.
The reason for this is that even if the dipole moment and the polarizability are linear for both $\alpha$ and $\beta$, in the $\mathrm{AH} 1$ and $\mathrm{AH} 2$ cases, these can cause optical transitions with the help of the anharmonic mode-mode couplings $g_{\alpha^{2} \beta} q_{\alpha}^{2} q_{\beta}$ and $g_{\alpha \beta^{2}} q_{\alpha} q_{\beta}^{2}$, which induce the double quantum transitions represented by the green double circles in Fig. 4, in addition to the zero and double quantum transitions for both $\alpha$ and $\beta$, as discussed in Ref. 19.

For the low-frequency modes depicted in Figs. 3(b-i) and 3(b-ii), we are not able to identify some of the peaks discussed above with regard to the high-frequency modes arising from the MAHC. This is due to the fact that these peaks overlap because their resonant frequency are small, while their widths are large. In Fig. 3(b-iii), it is seen that clear cross peaks (labeled by "D" and "E") arise from the EAHC in the NL2 case. In the NL3 case, however, the peaks labeled by "F" and "G" are indistinguishable, as are those labeled by " $\mathbf{F}^{\prime \prime}$ " and " $\mathbf{G}$ '," because the peaks at $\omega_{2}=\omega_{\alpha}+\omega_{\beta}$ and $\omega_{2}=\omega_{\alpha}-\omega_{\beta}$ overlap for small $\omega_{\beta}$.

\section{FULL MD SIMULATION APPROACH FOR TWO-DIMENSIONAL SPECTROSCOPIES}

\section{A. Equilibrium-nonequilibrium hybrid MD approach}

In 1D vibrational spectroscopic approaches employing full MD simulations, we can evaluate the observables easily by calculating optical properties from an ensemble of molecular trajectories. As illustrated in Eq. (15), the linear absorption (1D IR) spectrum, $I_{\mathrm{IR}}^{(1)}(\omega)$, and the 1D Raman spectrum, $I_{\text {Raman }}^{(3)}(\omega)$, are calculated from the response function in time domain, expressed as

$$
R\left(t_{1}\right)=\frac{1}{k_{B} T}\left\langle\boldsymbol{B}_{\mathrm{eq}}\left(t_{1}\right) \dot{\boldsymbol{A}}_{\mathrm{eq}}(0)\right\rangle .
$$
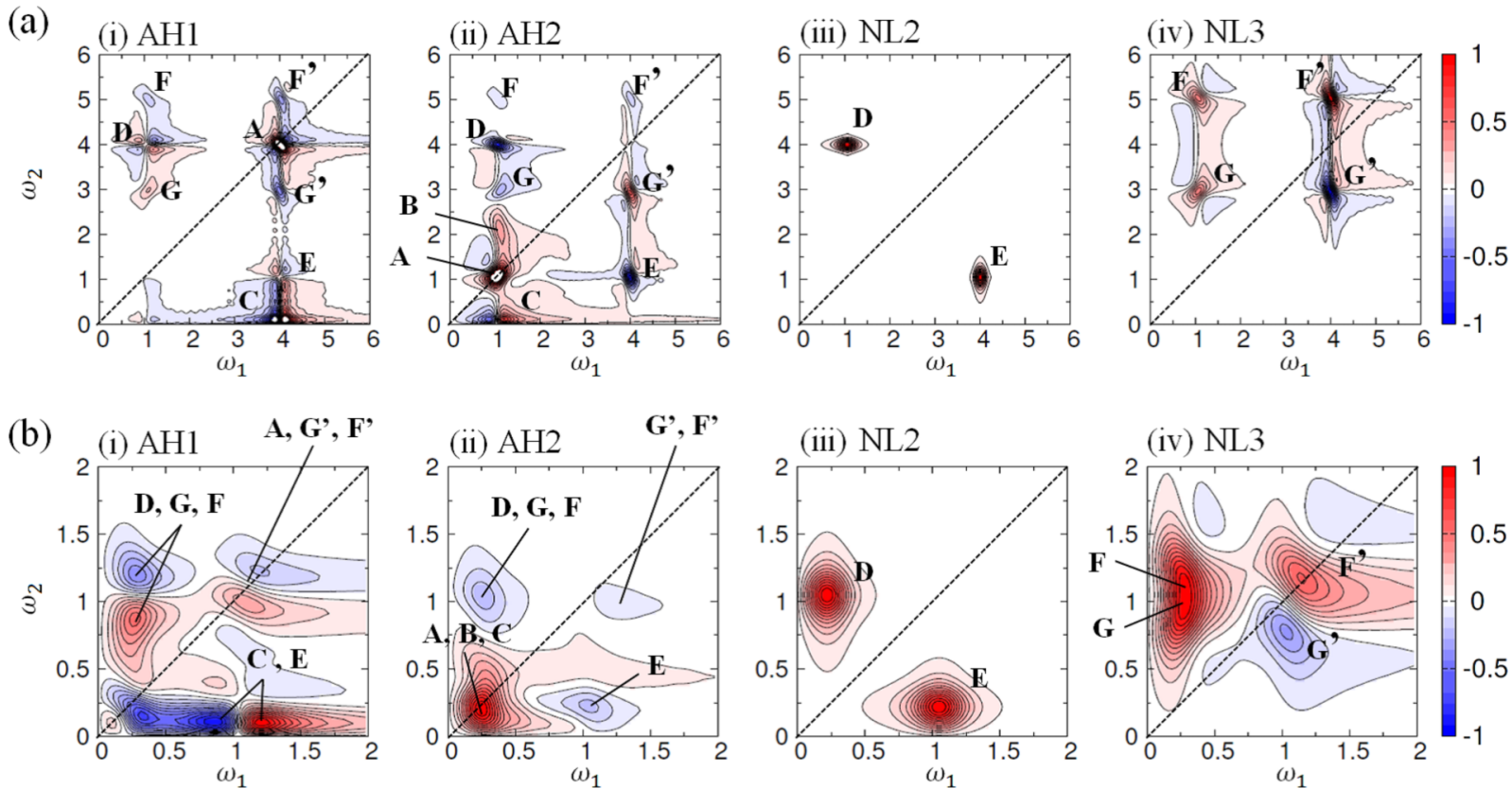

(iii) NL2

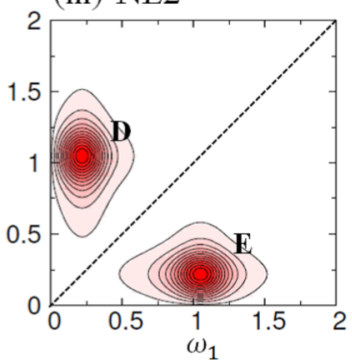

(iv) NL3

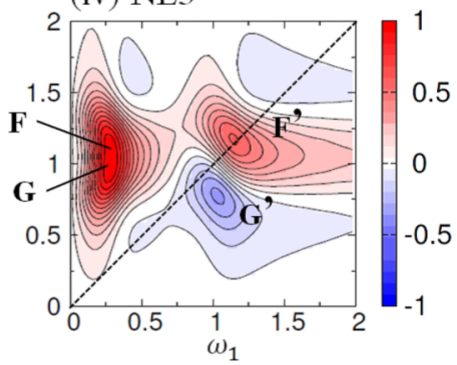

FIG. 3. 2D spectra in the (i) AH1, (ii) AH2, (iii) NL2 and (iv) NL3 cases. The spectra in the AH1 and AH2 cases arise from the MAHC, while those in the NL2 and NL3 cases arise from EAHC. These results were calculated using the two-mode SL BO model. The cases (a) and (b) are those with high-frequency and lowfrequency modes, respectively. The spectral intensities are all normalized. 
(a) NL2 contribution

(i)

(ii)

(iii)

(iv)

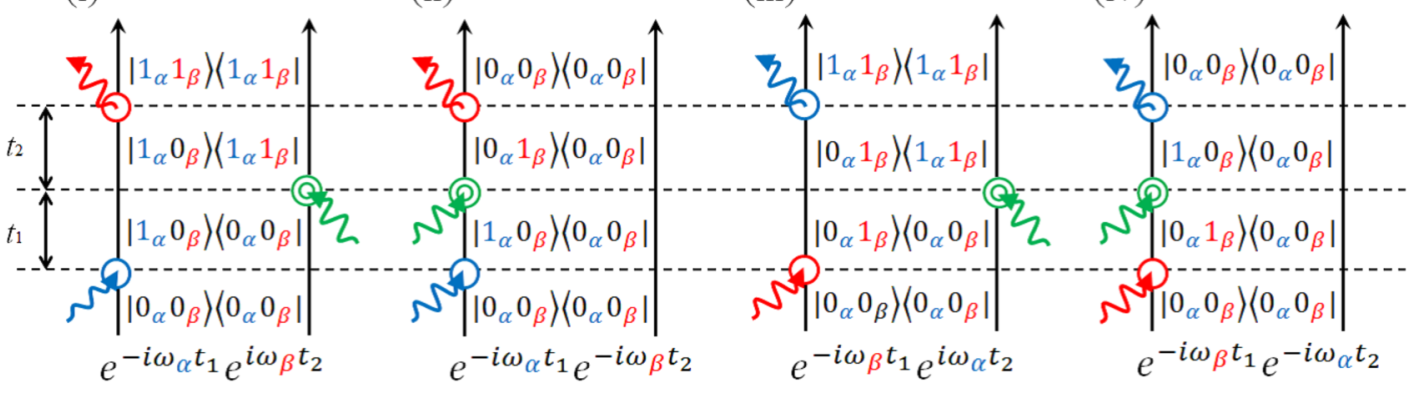

(b) NL3 contribution

(i)

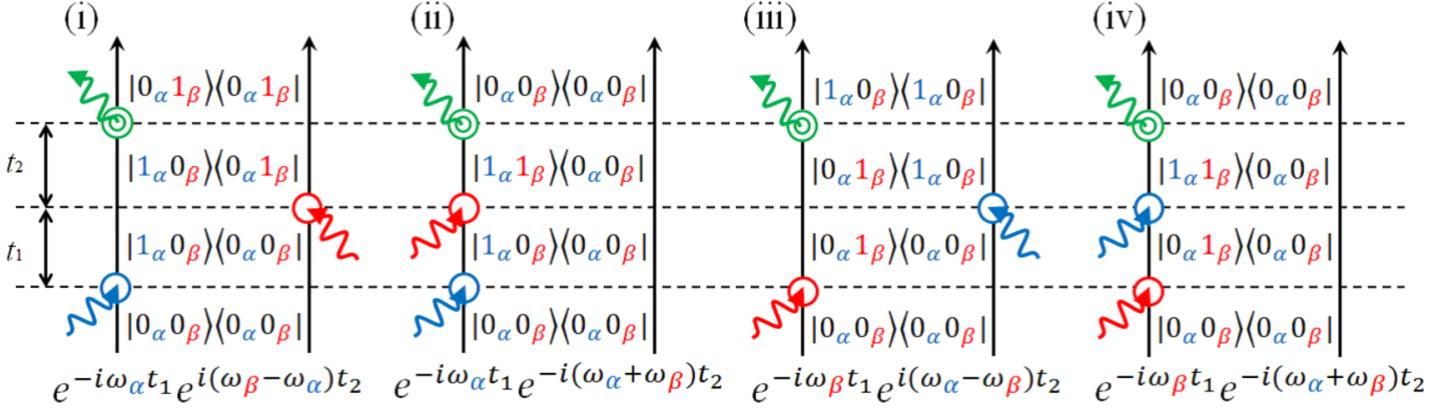

FIG. 4. The Liouville paths involved in the (a) NL2 and (b) NL3 cases for the mode $\alpha$ with frequency $\omega_{\alpha}$ and the mode $\beta$ with frequency $\omega_{\beta}$ are depicted. Here, an energy eigenstate of the harmonic potential is denoted by $\left|n_{\alpha}, n_{\beta}\right\rangle$, and we have depicted cases starting from the vibrational ground state. The blue circles and the red circles represent single quantum transitions with respect to mode $\alpha$ and mode $\beta$, whereas the green double circles represent double quantum transition with respect to both $\alpha$ and $\beta$ modes. The other paths, which are the Hermitian conjugates of the above paths, are not presented.

Here, $\boldsymbol{A}_{\mathrm{eq}}(t)$ and $\boldsymbol{B}_{\mathrm{eq}}(t)$ are the dipole moment or polarizability obtained from the equilibrium MD (EMD) trajectories at time $t$. The time derivative of $\boldsymbol{A}(t)$ is defined as $\dot{\boldsymbol{A}}_{\text {eq }}(0)$ $\equiv d \boldsymbol{A}_{\mathrm{eq}}(t) /\left.d t\right|_{t=0}$.

To calculate $2 \mathrm{D}$ signals, we apply the equilibrium-nonequilibrium hybrid MD algorithm originally developed for $2 \mathrm{D}$ Raman spectroscopy. ${ }^{16-18}$ The response functions in the cases of 2D IR-Raman and 2D Raman spectroscopy are evaluated as

$$
R\left(t_{2}, t_{1}\right)=\frac{1}{k_{B} T F \Delta t}\left\langle\dot{\boldsymbol{A}}_{\mathrm{eq}}\left(-t_{1}\right)\left(\boldsymbol{C}_{+\boldsymbol{B}(0)}\left(t_{2}\right)-\boldsymbol{C}_{-\boldsymbol{B}(0)}\left(t_{2}\right)\right)\right\rangle,
$$

where $\Delta t$ is the time step used in integrating the equations of motion, and $F$ is the constant that arises from the nonequilibrium MD (NEMD) perturbation. In the 2D RII, IRI, IIR, and 2D Raman cases, $F$ is given by $2 E_{1}, E_{1} E_{2}, 2 E_{1}$, and $E_{1} E_{2}$, respectively. The RII signal, for example, is calculated as follows. We first obtain the time derivative of the polarizability, $\dot{\boldsymbol{\Pi}}_{\mathrm{eq}}\left(-t_{1}\right)$, from the equilibrium trajectories at time $t=-t_{1}$. Next, we evaluate the dipole moments $\boldsymbol{\mu}_{+\boldsymbol{\mu}(0)}\left(t_{2}\right)$ and $\boldsymbol{\mu}_{-\boldsymbol{\mu}(0)}\left(t_{2}\right)$ at time $t=t_{2}$ from the non-equilibrium trajectories, which are generated by a perturbation at time $t=0, \mp \boldsymbol{\mu}(0) \boldsymbol{E}_{1} \delta(t)$, resulting from the external electric field of the $j$ th pulse $\boldsymbol{E}_{j}$ acting on the dipole moment $\boldsymbol{\mu}(0)$. The RII signal is then evaluated as $\left\langle\dot{\boldsymbol{\Pi}}_{\mathrm{eq}}\left(-t_{1}\right)\left(\boldsymbol{\mu}_{+\boldsymbol{\mu}(0)}\left(t_{2}\right)-\boldsymbol{\mu}_{-\boldsymbol{\mu}(0)}\left(t_{2}\right)\right)\right\rangle /\left(2 k_{B} T E_{1} \Delta t\right)$.

\section{B. POLI2VS potential}

To calculate the 2D IR-Raman and 2D Raman spectra on the basis of MD simulations, we utilized a polarizable water model for intermolecular and intramolecular vibrational spectroscopy (POLI2VS). ${ }^{46}$ In this flexible water model, the charge-flow polarizability, permanent charge, dipole moment, quadrupole moment, and atomic dipole polarizability are expressed as functions of the intramolecular-geometry. ${ }^{46}$ These functions are evaluated from the computational results with $a b$ initio calculations employing the coupled-cluster single- and double-excitation (CCSD) method with the aug-ccpVQZ basis set and distributed multipole analysis (DMA). ${ }^{63}$ In order to evaluate the optical observables measured in spectroscopy experiments, we calculated the total dipole moment, $\boldsymbol{\mu}(t)=\boldsymbol{\mu}^{\mathrm{P}}(t)+\boldsymbol{\mu}^{\mathrm{I}}(t)$, and total polarizability, $\boldsymbol{\Pi}(t)$ $=\Pi^{\mathrm{P}}(t)+\Pi^{\mathrm{I}}(t)$, of the liquid water system in a $\mathrm{MD}$ simulation, where $\boldsymbol{\mu}^{\mathrm{P}}(t), \boldsymbol{\mu}^{\mathrm{I}}(t), \boldsymbol{\Pi}^{\mathrm{P}}(t)$, and $\boldsymbol{\Pi}^{\mathrm{I}}(t)$ are the permanent dipole moment, induced dipole moment, permanent polarizability, and induced polarizability, given by $\boldsymbol{\mu}^{\mathrm{P}}(t)$ $=\sum_{i} \boldsymbol{\mu}_{i}^{\mathrm{p}}, \quad \boldsymbol{\mu}^{\mathrm{I}}(t)=\sum_{i} \boldsymbol{\mu}_{i}^{\mathrm{i}}, \quad \boldsymbol{\Pi}^{\mathrm{P}}(t)=\sum_{i} \boldsymbol{\Pi}_{i}^{\mathrm{p}}$, and $\boldsymbol{\Pi}^{\mathrm{I}}(t)=\sum_{i} \boldsymbol{\Pi}_{i}^{\mathrm{i}}$, respectively. Here, $\boldsymbol{\mu}_{i}^{\mathrm{p}}, \boldsymbol{\mu}_{i}^{\mathrm{i}}, \boldsymbol{\Pi}_{i}^{\mathrm{p}}$, and $\boldsymbol{\Pi}_{i}^{\mathrm{i}}$ are the permanent molecular dipole moment, induced molecular dipole moment, permanent molecular polarizability, and induced molecular polarizability of water molecule $i$, which are obtained using the full-order charge-flow dipole-induced-dipole (CFDID) model. ${ }^{46}$ The details of the full-order CFDID polarizability model and the POLI2VS potential model are presented in the supplementary material. ${ }^{64}$ It should be noted that the full-order CFDID model that we used in the previous investigation of 2D THz-Raman spectroscopy employed a fixed geometry for the rigid-body water model, ${ }^{17}$ while here we used the original fullorder CFDID polarizability of the POLI2VS potential model; the permanent molecular polarizability and the permanent 
charge-flow polarizability depend on the intra-molecular structure of water molecules.

\section{Simulation details}

To carry out the MD simulations for liquid water, the interactions between the molecules were modeled with POLI2VS. In the MD simulations, each system consisted of 64 molecules in a cubic box with periodic boundary conditions. The interaction potentials containing the quadrupole moments were cut off smoothly at a distance equal to half the length of the system using a switching function, and the long-range charge-charge, charge-dipole, and dipole-dipole interactions were calculated with the Ewald summation employing tinfoil boundary conditions. ${ }^{65}$ The equations of motion were integrated using the velocity-Verlet algorithm with $\Delta t=0.25 \mathrm{fs}$. The volume and energy were fixed after the completion of the isothermal simulations carried out to establish equilibration. The conditions of the simulation were set such that the average density and temperature were $0.997 \mathrm{~g} / \mathrm{cm}^{3}$ and $300 \mathrm{~K}$, respectively. The 1D IR and Raman spectra were calculated from the equilibrium trajectory data, while the 2D IR-Raman and 2D Raman spectra were calculated with the equilibrium-non-equilibrium hybrid MD simulation algorithm.

\section{RESULTS AND DISCUSSION}

In this section, we present our results for the 2D IRRaman and 2D Raman spectra calculated from the MD simulations described in Sec. III. Then, we analyze the obtained spectra using the LL+SL BO model described in Sec. II. In order to compare the effect of the anharmonicity with respect to the potential for each mode and mode-mode coupling clearly, we employ the dimensionless coordinate $\tilde{q}_{s} \equiv\left(\omega_{s} / \omega_{0}\right) \bar{q}_{s}$. The renormalized Hamiltonian is presented in Appendix B. To parametrize the BO model, we employed the (1) intramolecular $\mathrm{OH}$-stretching, (2) intramolecular $\mathrm{HOH}-$ bending, (3) HB-intermolecular librational, and (4) HBintermolecular vibrational (translational) modes. In order to reproduce the profiles of the $1 \mathrm{D}$ and $2 \mathrm{D}$ spectra obtained from the MD simulations using the LL+SL BO model, we express the signals in terms of the single-mode and coupled-mode contributions as

$$
I\left(\omega_{1}\right)=\sum_{s} I_{s}\left(\omega_{1}\right)
$$

and

$$
I\left(\omega_{2}, \omega_{1}\right)=\sum_{s} I_{s}\left(\omega_{2}, \omega_{1}\right)+\sum_{s, s^{\prime}>s} I_{s s^{\prime}}\left(\omega_{2}, \omega_{1}\right),
$$

where $I_{s}\left(\omega_{1}\right)$ and $I_{s}\left(\omega_{2}, \omega_{1}\right)$ are the $1 \mathrm{D}$ and $2 \mathrm{D}$ spectra arising from the single mode, $s$, whereas $I_{s s^{\prime}}\left(\omega_{2}, \omega_{1}\right)$ is the $2 \mathrm{D}$ spectrum arising from the coupled mode, $s$ and $s^{\prime}$.

The resonant frequency, the bath parameter values, and the linear optical properties of these modes were estimated from the 1D Raman and 1D IR spectra obtained from the MD simulations. The values of anharmonicity and nonlinearly of the dipole moment and/or polarizability were determined by requiring that the $2 \mathrm{D}$ spectra reproduce each of the $2 \mathrm{D}$ signal profiles obtained from the MD simulations by capturing their characteristic features. The calculations for the single- and coupled-mode spectra $I_{s}\left(\omega_{1}\right)$ and $I_{s}\left(\omega_{2}, \omega_{1}\right)$ were carried out separately, following the procedure explained in Secs. II C 1 and II C 2. In the coupled-mode case, $I_{s s^{\prime}}\left(\omega_{2}, \omega_{1}\right)$, however, we ignored the cubic anharmonicity of the potential (i.e., the terms $g_{s^{3}} q_{s}^{3}$ and $g_{s^{\prime 3}} q_{s^{\prime 3}}$ ) when we calculated the contributions of the 2D signals in order to avoid double counting of the AH contributions that had been taken into account by the signal-mode contributions, $I_{s}\left(\omega_{2}, \omega_{1}\right)$. The fitted system and bath parameter values for the $2 \mathrm{D}$ spectra of liquid water are listed in Tables III and IV with $\omega_{0}=4000 \mathrm{~cm}^{-1}$ and $T=300 \mathrm{~K}$. The 1D spectra were also calculated using these parameter values without including the coupled-mode contributions, because the spectral intensities of these contributions are significantly weaker than the single-mode contributions. The qualitative features of the peak profiles in the $2 \mathrm{D}$ spectra obtained from the MD simulations are accurately, reproduced with the multi-mode LL+SL BO model. The set of fitting parameters for entire system are determined uniquely in order to best reproduce all of the complex profiles of the 1D and 2D spectra obtained from the MD simulations. This result indicates that this model based analysis captures the essential features of the intermolecular and intramolecular motion. As we show below, by utilizing the extracted model parameter values, we can analyze the anharmonic mode-mode coupling, the nonlinearity of the polarizability and dipole moment, relaxation phenomena, and the vibrational dephasing processes for each mode, even in the case that the 2D spectral peaks obtained from the MD simulation overlap or are unclear.

In Fig. 5, we display (a) the 1D IR and 1D Raman spectra obtained from the MD simulations and the LL+SL BO model, (b) the $2 \mathrm{D}$ MD results, and (c) the $2 \mathrm{D}$ fitted results from the LL+SL BO model for the (i) 2D RII, (ii) 2D IRI, (iii) 2D IIR, and (iv) 2D Raman cases of liquid water. It should be

TABLE III. The parameter values of the LL+SL BO model for the (1) intramolecular OH-stretching, (2) intramolecular HOH-bending, (3) HB-intermolecular librational, and (4) HB-intermolecular vibrational modes fitted to reproduce the 1D and 2D vibrational spectra of liquid water obtained from the full MD simulations. We set the fundamental frequency and temperature as $\omega_{0}=4000 \mathrm{~cm}^{-1}$, and $T=300 \mathrm{~K}$. The correlation times of the Gaussian-Markovian bath noise, $\tau_{s}=1 / \gamma_{s}$, are also presented.

\begin{tabular}{cccccccccccc}
\hline \hline$s$ & $\omega_{s}\left(\mathrm{~cm}^{-1}\right)$ & $\gamma_{s} / \omega_{0}$ & $\tau_{s}(\mathrm{fs})$ & $\tilde{\zeta}_{s}$ & $\tilde{V}_{L L}^{(s)}$ & $\tilde{V}_{S L}^{(s)}$ & $\tilde{g}_{s^{3}}$ & $\tilde{\mu}_{s}$ & $\tilde{\mu}_{s s}$ & $\tilde{\Pi}_{s}$ & $\tilde{\Pi}_{s s}$ \\
\hline 1 & 3520 & $2.5 \times 10^{-2}$ & 333 & 6.2 & 0 & 1.0 & $-5.0 \times 10^{-2}$ & 3.5 & $1.2 \times 10^{-2}$ & 3.5 & $2.5 \times 10^{-2}$ \\
2 & 1710 & $1.8 \times 10^{-2}$ & 463 & 1.2 & 0 & 1.0 & $-1.4 \times 10^{-2}$ & 1.4 & 0 & 0.47 & $-3.9 \times 10^{-2}$ \\
3 & 430 & $8.5 \times 10^{-2}$ & 98 & 8.3 & $3.4 \times 10^{-3}$ & 1.0 & $9.7 \times 10^{-2}$ & 18.6 & 0 & 2.1 & -0.83 \\
4 & 115 & 0.50 & 17 & 2.8 & $2.8 \times 10^{-3}$ & 1.0 & $9.7 \times 10^{-2}$ & 31.3 & 2.1 & 9.0 & 2.3 \\
\hline \hline
\end{tabular}


TABLE IV. The parameter values of the LL+SL BO model for the anharmonic mode-mode coupling fitted to reproduce the $2 \mathrm{D}$ vibrational spectra of liquid water obtained from the full MD simulations.

\begin{tabular}{lccll}
\hline \hline$s-s^{\prime}$ & $3 \tilde{g}_{s^{2} s^{\prime}}$ & \multicolumn{1}{c}{$3 \tilde{g}_{s s^{\prime 2}}$} & \multicolumn{1}{c}{$\tilde{\mu}_{s s^{\prime}}$} & \multicolumn{1}{c}{$\tilde{\Pi}_{s s^{\prime}}$} \\
\hline $1-2$ & $5.7 \times 10^{-2}$ & $5.7 \times 10^{-2}$ & $2.0 \times 10^{-3}$ & $2.6 \times 10^{-3}$ \\
$1-3$ & $-3.9 \times 10^{-2}$ & $-3.9 \times 10^{-2}$ & 0.13 & 0.19 \\
$1-4$ & $-7.5 \times 10^{-2}$ & $-7.5 \times 10^{-2}$ & 0.23 & 0.26 \\
$2-3$ & $-1.5 \times 10^{-2}$ & $-1.5 \times 10^{-2}$ & $6.3 \times 10^{-2}$ & $7.4 \times 10^{-2}$ \\
$2-4$ & $-2.0 \times 10^{-2}$ & $-2.0 \times 10^{-2}$ & $3.1 \times 10^{-2}$ & $3.1 \times 10^{-2}$ \\
$3-4$ & 0.23 & 0.23 & $7.8 \times 10^{-2}$ & 0.16 \\
\hline \hline
\end{tabular}

noted that because our BO model does not distinguish the IR active and Raman active modes, we could not fit the 1D Raman spectrum accurately using the same parameter values for the 1D IR spectrum. For each mode, the correlation time of the Gaussian-Markovian bath noise, $\tau_{s}=1 / \gamma_{s}$, is estimated as $\tau_{1}=334, \tau_{2}=463, \tau_{3}=98$ and $\tau_{4}=17$ (fs), respectively. These values are consistent with the time scale evaluated from experiments and MD simulations. ${ }^{4,36-43,67,68}$ From the
BO analysis, we found that the spectral peak labeled by "A" in the 2D IRI spectrum in Fig. 5(b-ii) arises from the HBintermolecular libration. This motion also yields the overtone peaks labeled by "B" in the 2D IIR spectrum Fig. 5(b-iii) as the NL3 contribution. A negative spectral peak labeled by "C" in the 2D IRI spectrum in Fig. 5(b-ii) represents the NL2 contribution of the HOH-bending motion. It is seen that the peak in the 2D IRI spectrum overlaps with that resulting from the MAHC contribution involving the $\mathrm{OH}$-stretching mode and $\mathrm{HOH}$-bending mode, labeled by "D." The overtone peak of the HOH-bending mode in the 2D IIR spectrum displayed in (b-iii) as the NL3 contribution is weak, and thus the spectral peak labeled by ' $\mathbf{D}$ '," which results from the MAHC contribution involving the $\mathrm{OH}$-stretching mode and $\mathrm{HOH}$-bending mode, is more visible.

The peaks corresponding to the OH-stretching motion, labeled by "E" in the 2D IR-Raman and 2D Raman spectra, arise from the anharmonic contribution due to the strong anharmonicity of the $\mathrm{OH}$-stretching motion. There also exist overtone peaks of the $\mathrm{OH}$-stretching motion near $\left(\omega_{1}, \omega_{2}\right)=(4000,7000) \mathrm{cm}^{-1}$ in both the MD and BO results,

(a) 1D Spectra
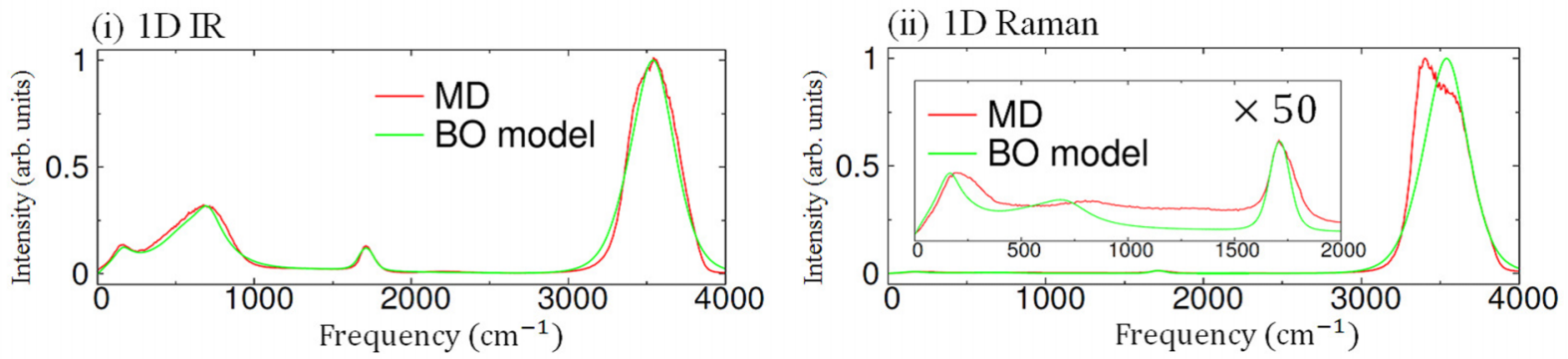

(b) MD Simulation

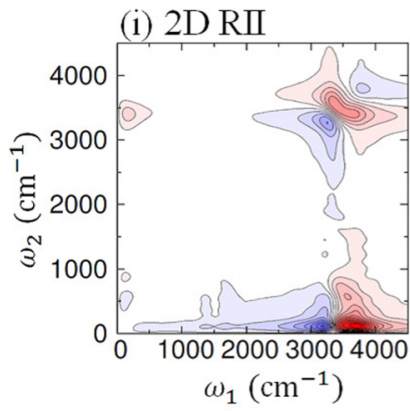

(ii) $2 \mathrm{D}$ IRI

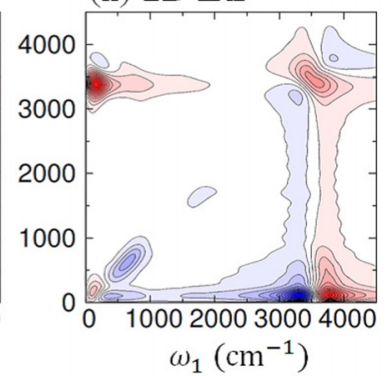

(iii) $2 \mathrm{D} \Pi \mathrm{IR}$

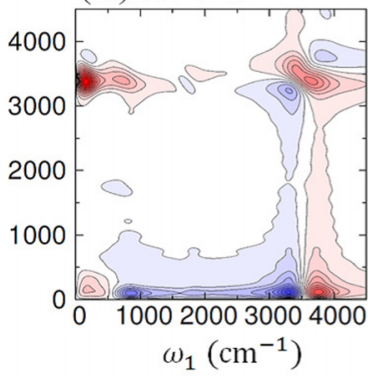

(iv) $2 \mathrm{D} R \mathrm{RR}$

(c) $\mathrm{BO}$ model

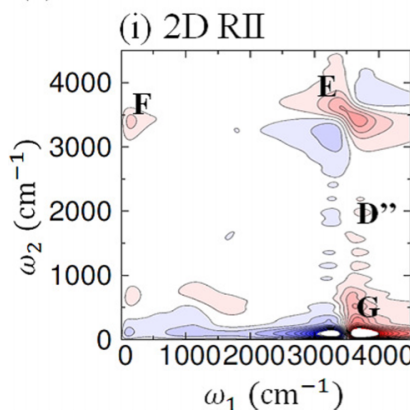

(ii) $2 \mathrm{D}$ IRI

(iii) $2 \mathrm{D}$ IIR

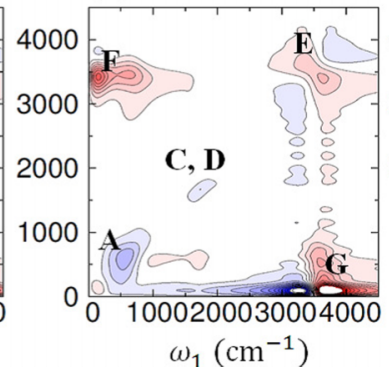

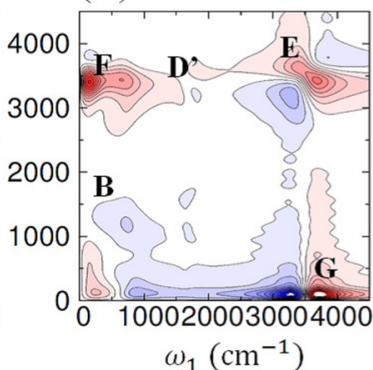

(iv) $2 \mathrm{D} R R R$

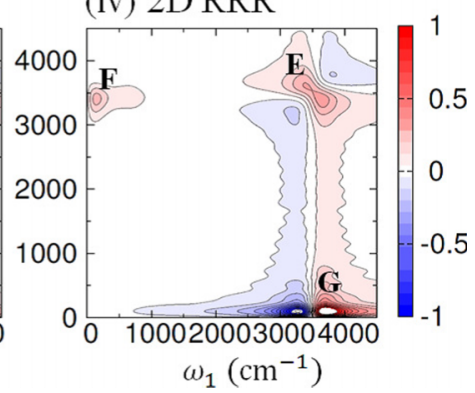

FIG. 5. (a) The 1D signals of (i) IR and (ii) Raman for liquid water obtained from the MD simulations (red curves) and the LL+SL BO model (green curves). (b) The 2D signals obtained from the MD simulations. (c) The 2D signals obtained from the LL+BO model. In (b) and (c), (i), (ii), and (iii) display the results for the $z, z, z$ tensor element of the 2D RII, 2D IRI and 2D IIR spectra, respectively, while (iv) displays the results for the $z z z z z z$ tensor element of the 2D Raman spectrum. The spectral intensities are all normalized. 
but these are outside the frequency range of Fig. 5. (See Fig. 9 in Appendix $\mathrm{C}$ for a plot of the MD case.) The overtone peaks of the $\mathrm{OH}$-stretching and $\mathrm{HOH}$-bending motion, which correspond to the doubly excited peaks in the quantum picture, are caused by the nonlinearity of the optical dipole or the polarizability. The cross peaks labeled by "F" in all of the 2D spectra arise from the interaction between the intermolecular modes and intramolecular $\mathrm{OH}$-stretching mode: These peaks are prominent in comparison with the other mode-mode coupling peaks, because the anharmonic elements for these peaks are much larger than those for the other peaks, as seen in Table IV. It should be noted that the cross peaks labeled by "B," "D'," and "G" in Fig. 5(b) appear at the peak positions for the libration-bending, bending-stretching, and translation-stretching mode-mode coupling, respectively. However, they may also contain contributions from the overtone and population relaxation processes involved in the NL3 and AH contributions, as illustrated in Fig. 2. In Appendix C, we further analyze the MD results in terms of three contributions, those of the permanent dipole moment and polarizability, the induced dipole moment and polarizability, and a mixture of the permanent and induced dipole moment and polarizability. This is done in order to elucidate the roles of the optical properties for each of the modes.

To investigate the mechanism of the intermolecular HB and intramolecular $\mathrm{OH}$-stretching coupling further, we enlarge the spectra near the peak labeled by "F" in Fig. 6. Moreover, in Fig. 7, we plot the contribution of the HB-intermolecular vibrational and $\mathrm{HB}$-intermolecular librational modes in the MAHC and EAHC cases separately, in order to analyze the origin of the signal. Note that here we consider the 2D IRI case, but because our BO model does not distinguish between the IR and Raman active modes, the MAHC and EAHC contributions in all of the 2D IR-Raman and 2D Raman spectra exhibit similar profiles; the only difference is in the relative intensities of the MAHC and EAHC contributions.

As illustrated in Fig. 7, in the MAHC case, we observe a negative peak and a positive peak whose node lines are centered at the resonant frequency. In the EAHC case, we observe a positive peak at the resonant frequency. The existence of the negative peaks labeled by "A" in Fig. 7 indicates a strong anharmonic coupling between the $\mathrm{OH}-$ stretching mode and the HB-intermolecular vibrational modes. The peaks labeled by "B" involve both the MAHC and EAHC contributions. The small peaks labeled by " $C$ " in the IRI and IIR spectra displayed in Figs. 6(ii) and 6(iii) arise from the EAHC interaction between the $\mathrm{OH}$-stretching mode and the HB-intermolecular librational modes. We do not observe these EAHC cross peaks in the 2D RII and 2D Raman spectra displayed in Figs. 6(i) and 6(iv), because in these cases, the HB-intermolecular librational motion could not be excited by the first Raman pulse in the RII and RRR processes, due to the weak polarizability of the librational motion.

The estimated values for the anharmonic coupling strength presented in Table IV indicate that the coupling strength between the $\mathrm{OH}$-stretching modes and the HBintermolecular vibrational modes is comparable to that between the $\mathrm{OH}$-stretching modes and $\mathrm{HOH}$-bending modes. This result is consistent with the results of analysis based on MD simulations. ${ }^{4,6}$ Moreover, the coupling between the $\mathrm{OH}$-stretching and HB-intermolecular vibrational modes can be measured experimentally by observing the negative cross peak between these modes, labeled by "A" in our figures. This finding of the possibility to measure the strength of the coupling between the $\mathrm{OH}$-stretching and HB-intermolecular vibration is important, because this coupling may be a cause

\section{(a) MD Simulation}

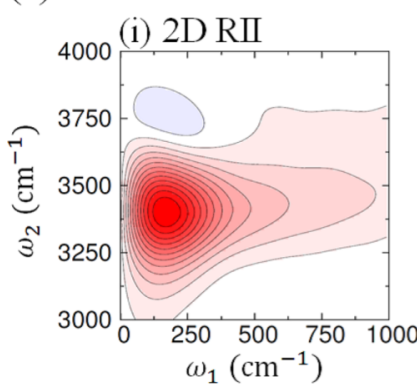

(b) $\mathrm{BO}$ model

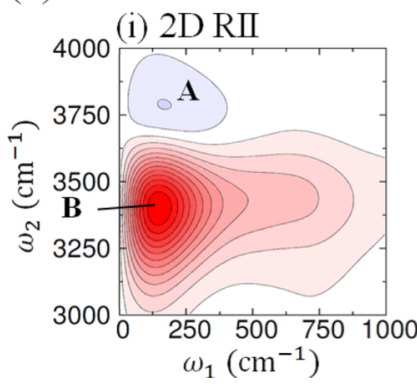

(ii) $2 \mathrm{D}$ IRI

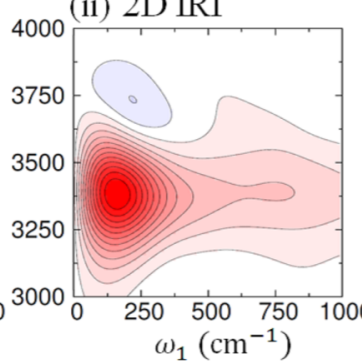

(ii) $2 \mathrm{D} I R I$

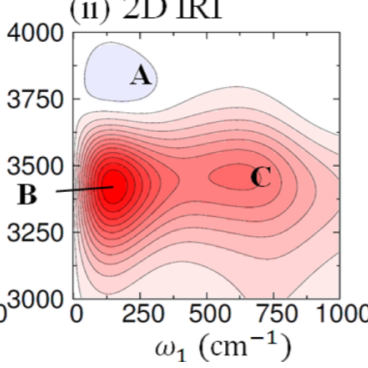

(iii) $2 \mathrm{D} \mathrm{IIR}$

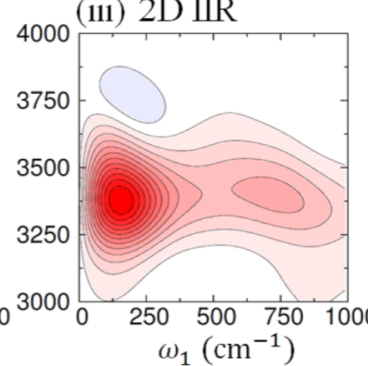

(iii) $2 \mathrm{D} \Pi \mathrm{IR}$

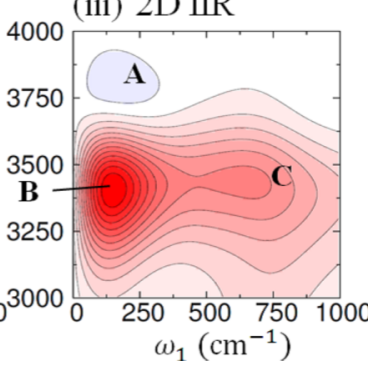

(iv) $2 \mathrm{D} R \mathrm{RRR}$

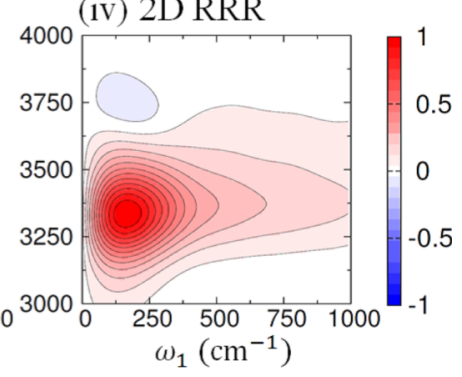

(iv) $2 \mathrm{D} R \mathrm{RRR}$

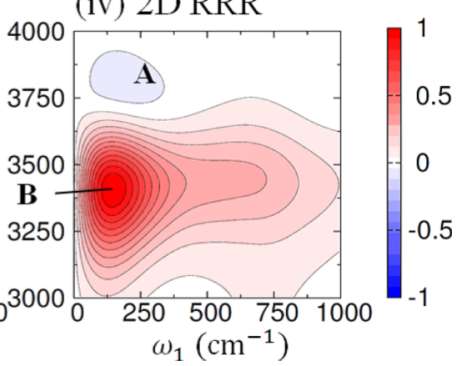

FIG. 6. (a) Results for the contributions of the intermolecular HB and intramolecular OH-stretching mode-mode coupling obtained from MD simulations. (b) The corresponding results obtained from BO model calculations. (i), (ii) and (iii) display the results for the $z z z z$ tensor element of the 2D RII, 2D TRI and 2D TIR spectra, respectively, while (iv) displays the results for the $z z z z z z$ tensor element of the 2D Raman spectrum. The spectral intensities are all normalized with respect to the absolute value of the spectral peak intensities. 
(a) HB-intermolecular vibration
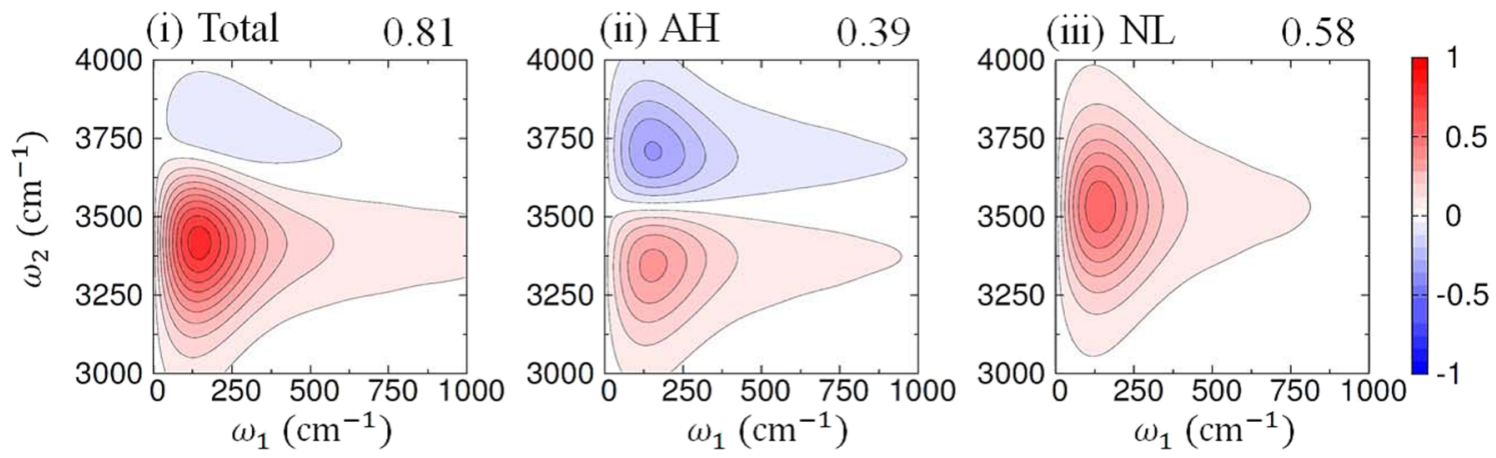

(b) HB-intermolecular libration
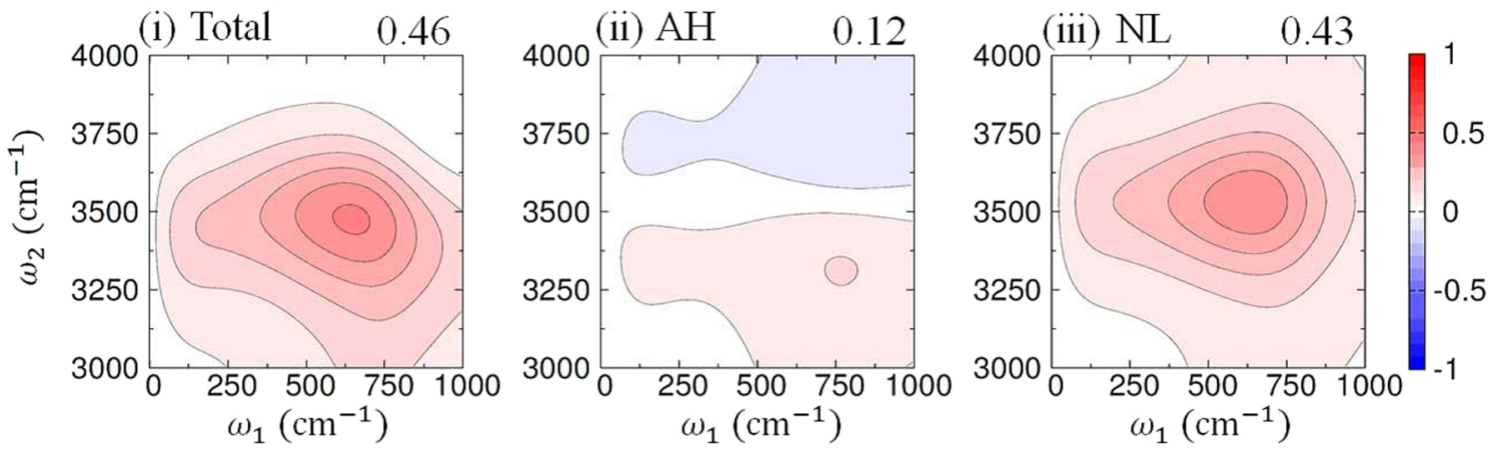

\section{(c) HB-intermolecular vibration and libration}
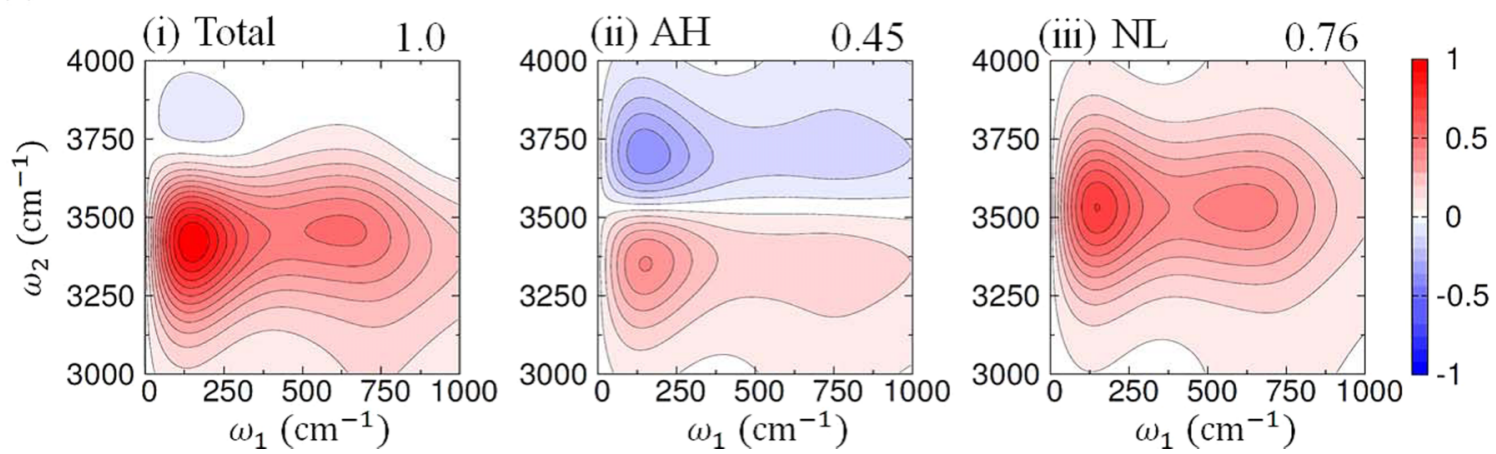

FIG. 7. The 2D IRI spectrum decomposed into (a) the OH-stretching and HB-intermolecular vibrational coupling modes, (b) the OH-stretching and HB-intermolecular librational coupling modes, and (c) the OH-stretching, HB-intermolecular vibrational and HB-intermolecular librational modes. The results displayed here are for the (i) MAHC+EAHC, (ii) MAHC, and (iii) EAHC cases calculated using the LL+SL BO model. The spectral intensities are all normalized. Also, we display the relative intensities with respect to the 2D IRI case depicted in Fig. 6(b-ii) in the top-right side of each panel.

of the fast thermal relaxation of the OH-stretching mode observed experimentally. ${ }^{41}$

\section{CONCLUSION}

We calculated 2D IR-Raman and 2D Raman spectra for liquid water using the full classical MD simulation approach. The obtained 2D spectra exhibit diagonal and off-diagonal peaks corresponding to the $\mathrm{OH}$-stretching, $\mathrm{HOH}$-bending, HB-intermolecular librational and HB-intermolecular vibrational modes and their mode-mode couplings that arise from the MAHC and EAHC processes. The calculated signals were then analyzed with the four-mode LL+SL BO model to investigate the roles of intermolecular and intramolecular mode-mode interactions. We showed that the coupling strength between the $\mathrm{OH}$-stretching and HB-intermolecular vibrational modes is comparable to that between the $\mathrm{OH}$ stretching and $\mathrm{HOH}-b e n d i n g$ modes. Moreover, the coupling between the $\mathrm{OH}$-stretching and $\mathrm{HB}$-intermolecular vibrational mode can be observed as positive and negative peaks in 2D IR-Raman and 2D Raman spectra. Contrastingly, other mode-mode coupling peaks are not easy to separate from the overtone and population relaxation contributions arise from the EAHC. This finding of the possibility to measure the strength of the $\mathrm{OH}$-stretching and HB-intermolecular vibrational coupling is important, because this coupling may be a cause of the fast thermal relaxation of the $\mathrm{OH}$ stretching mode. While no single light source can cover both intermolecular and intramolecular modes at the moment, 2D IR-Raman measurements may provide a practical method to explore the roles of intramolecular and intermolecular modes in liquid water. Although it is not easy to experimentally 
measure 2D IR-Raman signals, the peak positions predicted by the present analysis should be helpful in designing such experiments.

In this work, we employed the POLI2VS potential model $^{46}$ to calculate the 2D signal. Although this potential can reproduce 1D Raman and 1D IR signals for intermolecular and intermolecular modes reasonably well, the force field potential is not sufficiently accurate to obtain the correct profiles for the 2D IR-Raman and 2D Raman signals, due to the sensitivity of the nonlinear response function. ${ }^{17}$ Moreover, while a quantum mechanical treatment of the intramolecular mode is important for 2D vibrational spectroscopy, ${ }^{49,69-71}$ the present results and analysis are limited to the classical case. While the quantum MD simulation of liquid water is difficult because it requires a great deal of CPU power, a more practical approach may be to calculate 2D spectra quantum mechanically on the basis of the multi-mode LL+SL BO model with the set of parameter values obtained in the present study, applied through use of quantum hierarchal Fokker-Planck equations. ${ }^{48-60}$ The capability of quantum mechanical model calculations for 2D spectra also provides the possibility to directly analyze experimentally obtained spectra instead of classical MD simulation results. The LL+SL BO model developed in this work can be applied to the investigation of energy and excitation transfer process of liquid water. Extensions of the present treatment to 2D IR spectroscopy defined in terms of third-order response functions should also be possible. We leave such extensions to future studies, in accordance with progress in experimental and simulational techniques.

\section{ACKNOWLEDGMENTS}

The authors would like to thank Dr. Taisuke Hasegawa for providing the EMD source code for the POLI2VS potential model. Y.T. is thankful to professor Shinji Saito and Dr. Yuki Nagata for helpful discussions. This research is supported by a Grant-in-Aid for Scientific Research (No. A26248005) from the Japan Society for the Promotion of Science. A part of the computational resources used in this work was provided by the Research Center for Computational Science, Okazaki, Japan.

\section{APPENDIX A: CLASSICAL HIERARCHAL FOKKER-PLANCK EQUATIONS IN THE HERMITE REPRESENTATION}

The hierarchal Fokker-Planck equations, Eq. (8), converge very slowly as difference equations with a discrete mesh in phase space. The convergence can be improved by expanding $W^{\left(n_{1}, n_{2}\right)}(\boldsymbol{p}, \boldsymbol{q} ; t)$ in terms of Hermite functions in the momentum direction as ${ }^{19,72}$

$$
\begin{aligned}
W^{\left(n_{1}, n_{2}\right)}(\boldsymbol{p}, \boldsymbol{q} ; t)= & \psi_{0}\left(p_{\alpha}\right) \psi_{0}\left(p_{\beta}\right) e^{-\beta U_{\boldsymbol{\alpha}+\beta}(\boldsymbol{q}) / 2} \\
& \times \sum_{k=0}^{\infty} \sum_{l=0}^{\infty} c_{k, l}^{\left(n_{1}, n_{2}\right)}(\boldsymbol{q} ; t) \psi_{k}\left(p_{\alpha}\right) \psi_{l}\left(p_{\beta}\right),
\end{aligned}
$$

where $U_{\alpha+\beta}(\boldsymbol{q}) \equiv U_{\alpha}\left(q_{\alpha}\right)+U_{\beta}\left(q_{\beta}\right)+U_{\alpha \beta}\left(q_{\alpha}, q_{\beta}\right)$ is the potential of the system and $\psi_{j}\left(p_{s}\right)$ is the $j$ th Hermite function,

$$
\psi_{j}\left(p_{s}\right)=\frac{1}{\sqrt{2^{j} j ! a_{s} \sqrt{\pi}}} H_{j}\left(\frac{p_{s}}{a_{s}}\right) \exp \left(-\frac{p_{s}^{2}}{2 a_{s}^{2}}\right),
$$

with $H_{j}(x)$ the $j$ th Hermite polynomial and $a_{s}=\sqrt{2 m_{s} k_{B} T}$.

The coefficients $c_{k, l}^{\left(n_{1}, n_{2}\right)}(\boldsymbol{q} ; t) \equiv c_{k, l}^{\left(n_{1}, n_{2}\right)}\left(q_{\alpha}, q_{\beta} ; t\right)$ are expressed as ${ }^{19}$

$$
\begin{aligned}
\frac{\partial c_{k, l}^{\left(n_{1}, n_{2}\right)}(\boldsymbol{q} ; t)}{\partial t}= & \sqrt{k+1} \hat{D}_{\alpha}^{+} c_{k+1, l}^{\left(n_{1}, n_{2}\right)}(\boldsymbol{q} ; t)-\sqrt{k} \hat{D}_{\alpha}^{-} c_{k-1, l}^{\left(n_{1}, n_{2}\right)}(\boldsymbol{q} ; t) \\
& +\sqrt{l+1} \hat{D}_{\beta}^{+} c_{k, l+1}^{\left(n_{1}, n_{2}\right)}(\boldsymbol{q} ; t)-\sqrt{l} \hat{D}_{\beta}^{-} c_{k, l-1}^{\left(n_{1}, n_{2}\right)}(\boldsymbol{q} ; t) \\
& -n_{1} \gamma_{\alpha} c_{k, l}^{\left(n_{1}, n_{2}\right)}(\boldsymbol{q} ; t)-n_{2} \gamma_{\beta} c_{k, l}^{\left(n_{1}, n_{2}\right)}(\boldsymbol{q} ; t) \\
& -n_{1} \gamma_{\alpha} \Theta_{k+1}^{(\alpha)} c_{k+1, l}^{\left(n_{1}-1, n_{2}\right)}(\boldsymbol{q} ; t) \\
& -n_{2} \gamma_{\beta} \Theta_{l+1}^{(\beta)} c_{k, l+1}^{\left(n_{1}, n_{2}-1\right)}(\boldsymbol{q} ; t) \\
& -\Phi_{k-1}^{(\alpha)} c_{k-1, l}^{\left(n_{1}+1, n_{2}\right)}(\boldsymbol{q} ; t)-\Phi_{l-1}^{(\beta)} c_{k, l-1}^{\left(n_{1}, n_{2}+1\right)}(\boldsymbol{q} ; t),
\end{aligned}
$$

where

$$
\begin{aligned}
\hat{D}_{s}^{ \pm} & =\frac{1}{2} \frac{1}{\sqrt{m_{s} k_{B} T}} \frac{\partial U_{\alpha+\beta}(\boldsymbol{q})}{\partial q_{s}} \mp \frac{1}{m_{s}} \sqrt{m_{s} k_{B} T} \frac{\partial}{\partial q_{s}}, \\
\Theta_{j}^{(s)} & =-\zeta_{s} \frac{V^{(s)}\left(\boldsymbol{q}_{s}\right)}{\partial q_{s}} \sqrt{m_{s} k_{B} T} \sqrt{j},
\end{aligned}
$$

and

$$
\Phi_{j}^{(s)}=\frac{V^{(s)}\left(\boldsymbol{q}_{s}\right)}{\partial q_{s}} \frac{1}{\sqrt{m_{s} k_{B} T}} \sqrt{j+1} .
$$

We chose $k_{\max }$ and $l_{\max }$ so as to satisfy $c_{k, l} \approx 0 \quad(k$ $\geq k_{\max }$ or $l \geq l_{\max }$ ) and solved Eq. (A3) as $k_{\max }$ and $l_{\max }$ simultaneous equations. In this representation, the terminators of the equations of motion are obtained by replacing the operators as $\Phi_{k-1}^{(\alpha)} c_{k-1, l}^{\left(n_{1}+1, n_{2}\right)}(\boldsymbol{q} ; t) \rightarrow-\Phi_{k-1}^{(\alpha)} \Theta_{k}^{(\alpha)} c_{k, l}^{\left(n_{1}, n_{2}\right)}(\boldsymbol{q} ; t)$ and $\Phi_{l-1}^{(\beta)} c_{k, l-1}^{\left(n_{1}, n_{2}+1\right)}(\boldsymbol{q} ; t) \rightarrow-\Phi_{l-1}^{(\beta)} \hat{\Theta}_{l}^{(\beta)} c_{k, l}^{\left(n_{1}, n_{2}\right)}(\boldsymbol{q} ; t)$.

In the numerical calculations, we employed the dimensionless coordinate $\bar{q}_{s}$. The hierarchal coefficients are then defined as $\bar{c}_{k, l}^{\left(n_{1}, n_{2}\right)}\left(\bar{q}_{s}, \bar{q}_{s^{\prime}}, \bar{t}\right) \equiv\left(1 / \omega_{0} \sqrt{m_{s} k_{B} T}\right)^{n_{1}}$ $\left(1 / \omega_{0} \sqrt{m_{s^{\prime}} k_{B} T}\right)^{n_{2}} c_{k, l}^{\left(n_{1}, n_{2}\right)}\left(q_{s}, q_{s^{\prime}} ; t\right)$, where $\omega_{0}$ represents the fundamental frequency.

\section{APPENDIX B: DIMENSIONLESS HAMILTONIAN OF THE TWO-MODE SYSTEM}

In order to compare the effect of anharmonicity with respect to the potential for each mode and mode-mode coupling, we employed the dimensionless coordinates $\tilde{q}_{s}$ $\equiv\left(\omega_{s} / \omega_{0}\right) \bar{q}_{s}$. In this case, the system Hamiltonian of the two-mode BO model, given in Eq. (26), is expressed as

$$
\begin{aligned}
H_{s+s^{\prime}}\left(\tilde{q}_{s}, \tilde{q}_{s^{\prime}}\right)= & \hbar \omega_{0}\left\{\frac{1}{2}\left[\tilde{p}_{s}^{2}+\tilde{p}_{s^{\prime}}^{2}+\tilde{q}_{s}^{2}+\tilde{q}_{s^{\prime}}^{2}\right]\right. \\
& +\frac{1}{3 !}\left[\tilde{g}_{s^{3}} \tilde{q}_{s}^{3}+3 \tilde{g}_{s^{2} s^{\prime}} \tilde{q}_{s}^{2} \tilde{q}_{s^{\prime}}\right. \\
& \left.\left.+3 \tilde{g}_{s s^{\prime}} \tilde{q}_{s} \tilde{q}_{s^{\prime 2}}+\tilde{g}_{s^{\prime}} \tilde{q}_{s^{\prime}}^{3}\right]\right\},
\end{aligned}
$$



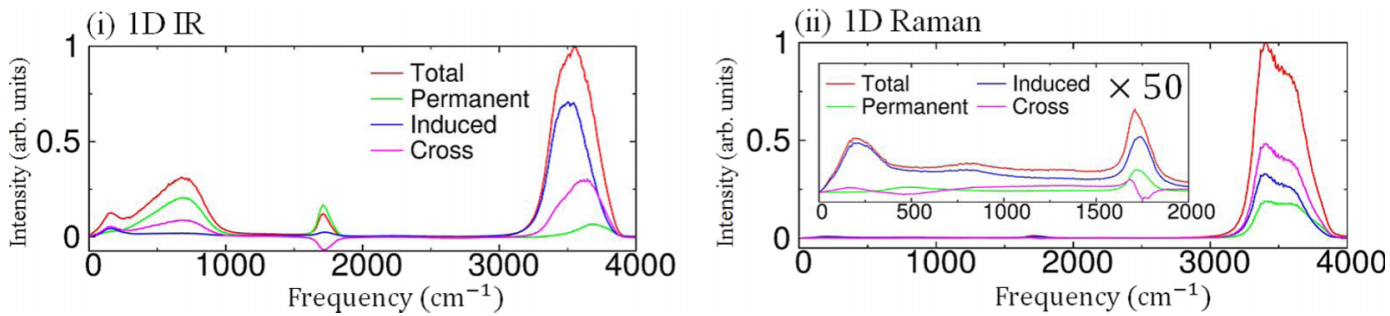

FIG. 8. (a) The 1D spectra of (i) IR and (ii) Raman for liquid water obtained from MD simulations expressed as the total (red), permanent (green), induced (blue), and cross (pink) contributions.

(a) Total

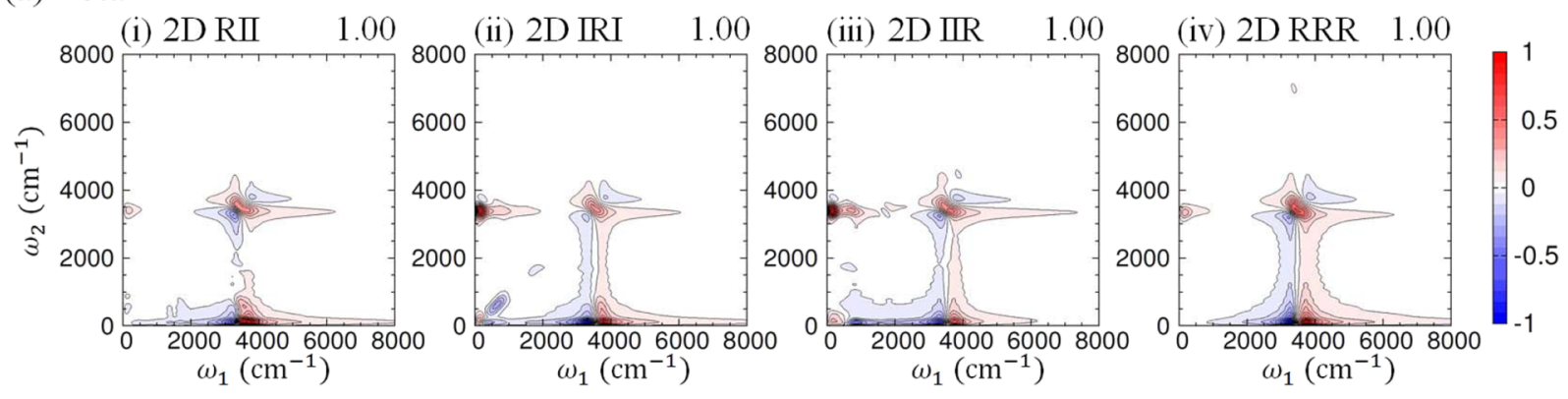

(b) Permanent
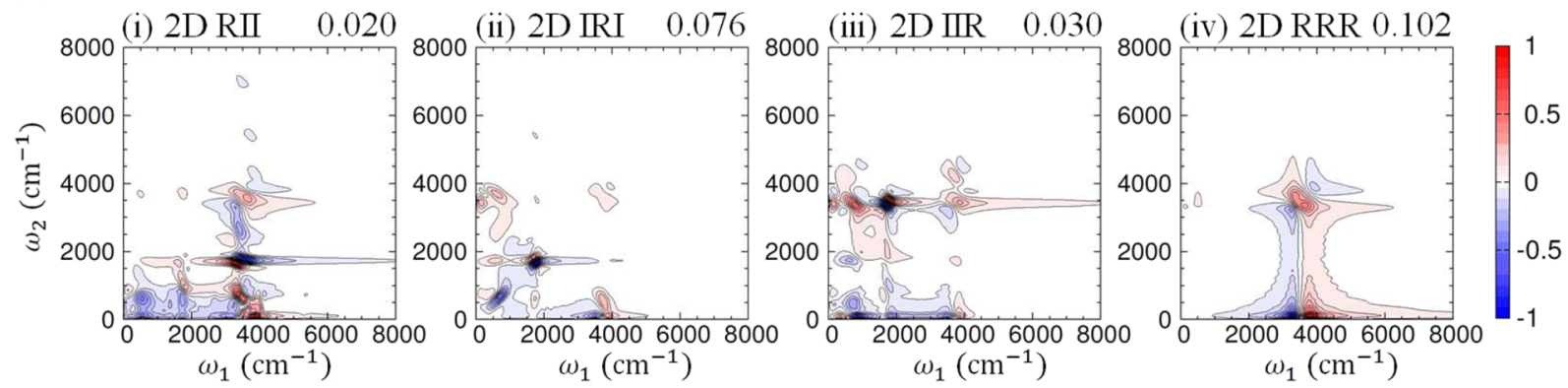

\section{(c) Induced}
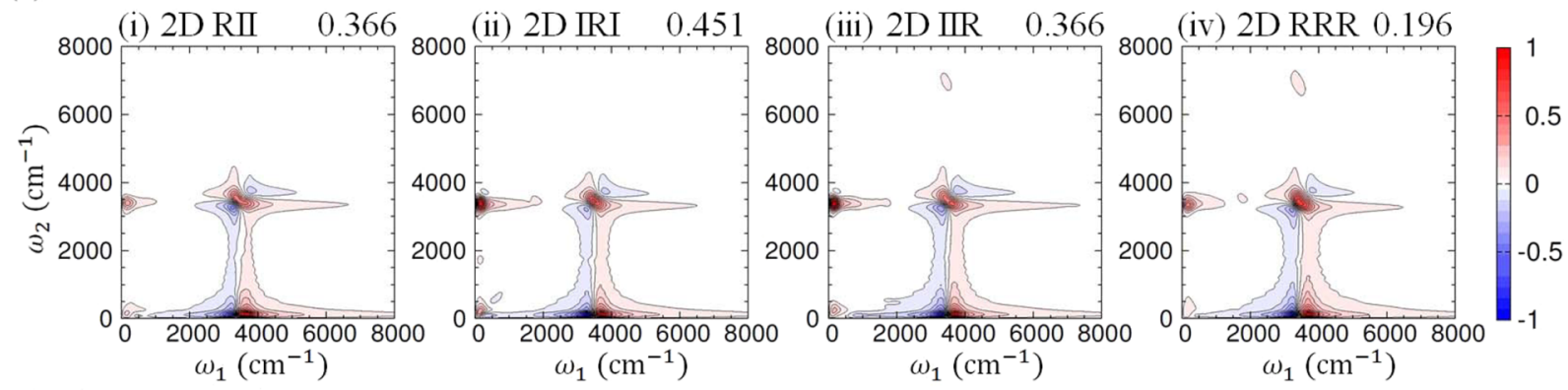

(d) Cross contribution
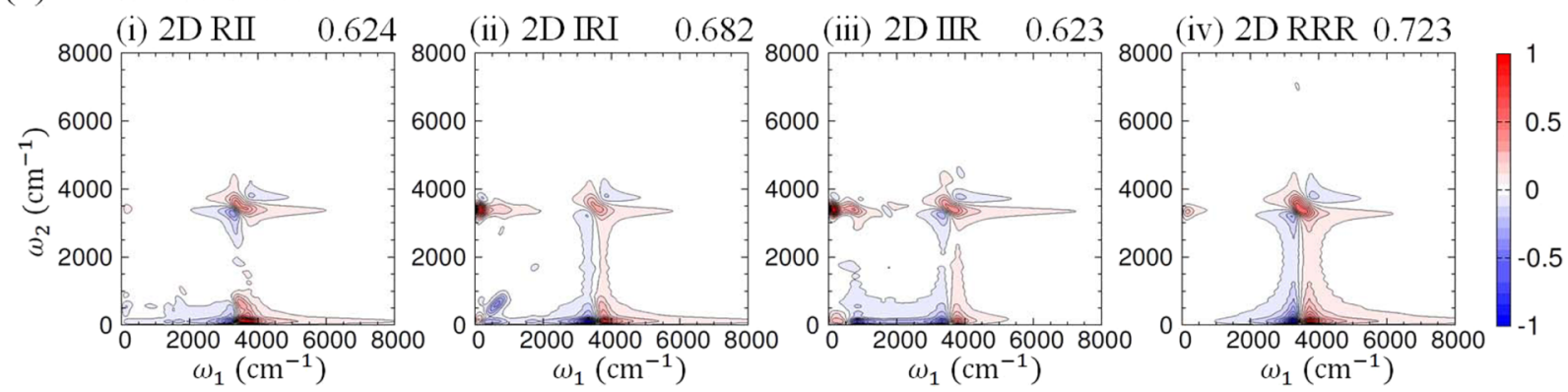

FIG. 9. The MD results for the $z, z z z$ tensor element of the (i) 2D RII, (ii) 2D IRI, and (iii) 2D IIR spectra and the $z, z, z z z$, tensor element of (iv) the 2D Raman spectra expressed as the (a) total (b) permanent, (c) induced, and (d) mixed contributions for liquid water. We display the relative intensities with respect to the total contribution of each 2D spectrum in the top-right side of each panel. The peaks near $\left(\omega_{1}, \omega_{2}\right)=(4000,7000) \mathrm{cm}^{-1}$ are the overtone peaks of the $\mathrm{OH}$-stretching motion. 
(a) 1D Spectra (Permanent)
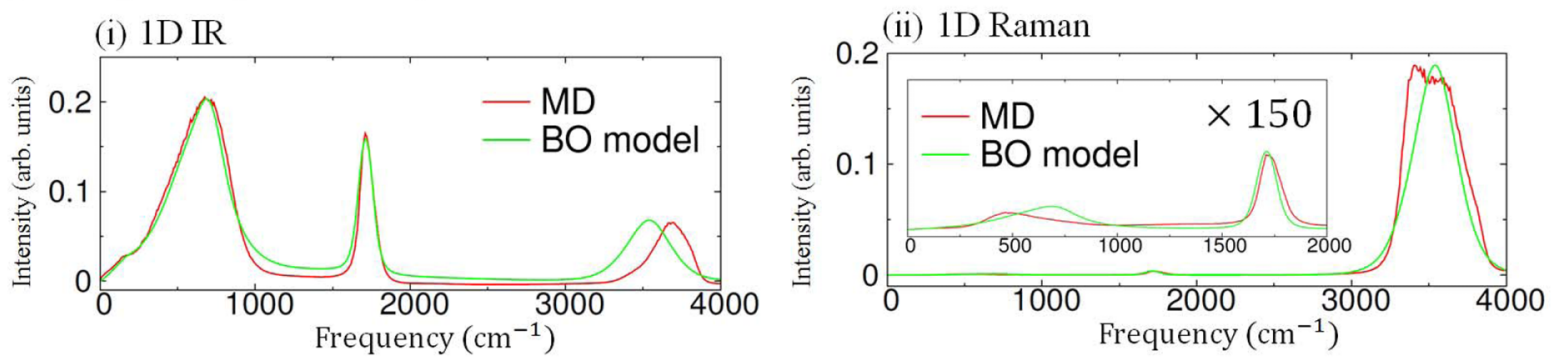

(b) MD Simulation (Permanent)

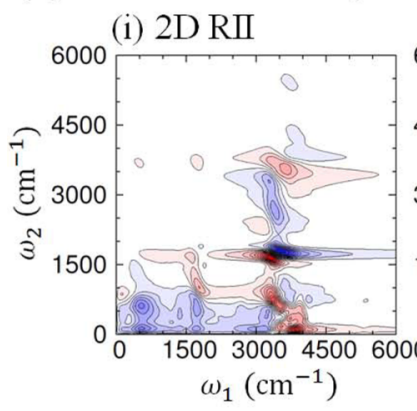

(ii) $2 \mathrm{D} \mathrm{IRI}$

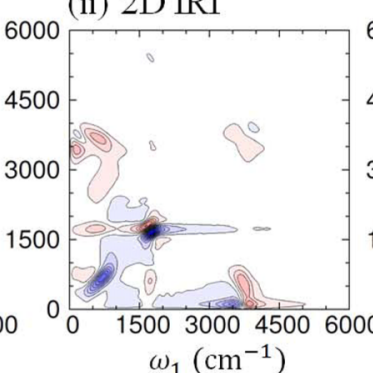

(iii) $2 \mathrm{D}$ IIR

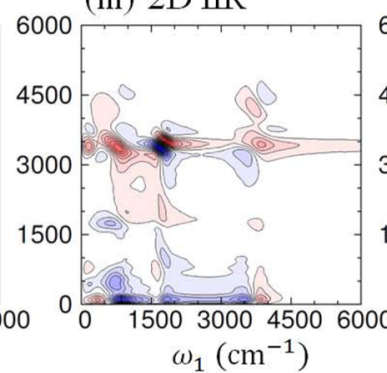

(iv) $2 \mathrm{D} R \mathrm{RR}$

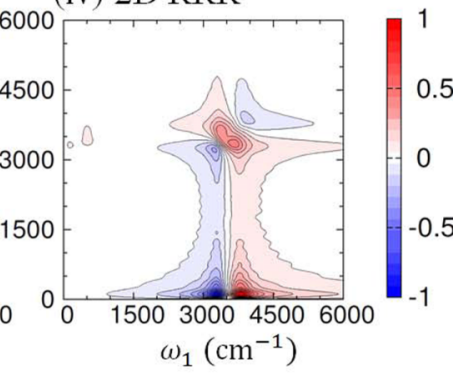

(c) $\mathrm{BO}$ model (Permanent)

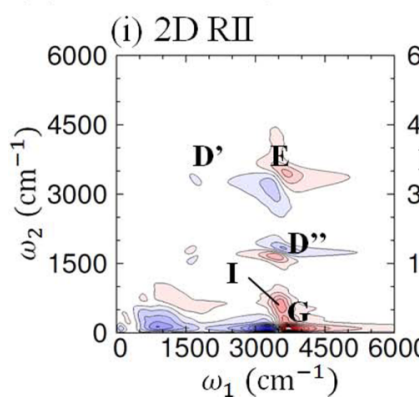

(ii) $2 \mathrm{D}$ IRI

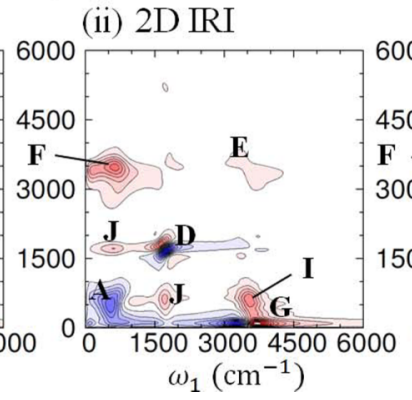

(iii) $2 \mathrm{D} \mathrm{IIR}$

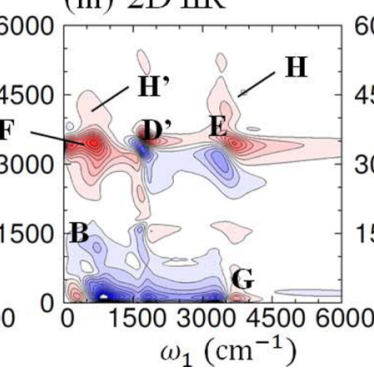

(iv) $2 \mathrm{D} \mathrm{RRR}$

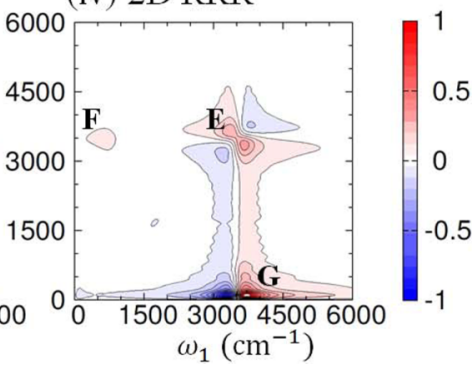

FIG. 10. (a) The permanent dipole and polarizability contributions to the 1D spectra obtained from the MD simulations (red curves) and LL+SL BO model (green curves). The results displayed in (i) and (ii) are the IR and Raman spectra, respectively. (b) The permanent contributions to the 2D spectra obtained from the MD simulation. (This is the same as Fig. 9(b).) (c) The permanent contributions to the 2D spectra obtained from the LL+BO model. In (b) and (c), the results displayed in (i), (ii) and (iii) are those for the $z z z z$ tensor element of the 2D RII, 2D IRI and 2D IIR spectra, while the results displayed in (iv) are those for the $z z z z z z$ tensor element of the Raman spectrum. All spectral intensities are normalized.

where $\tilde{g}$ is the strength of the anharmonicity. Using the dimensionless coordinate $\tilde{q}_{s}$, the system-bath coupling strength, LL coupling strength, SL coupling strength, and hierarchal coefficients are defined as $\tilde{\zeta}_{s} \equiv\left(\omega_{0} / \omega_{s}\right)^{2} \bar{\zeta}_{s}, \quad \tilde{V}_{L L}^{(s)} \equiv\left(\omega_{s} / \omega_{0}\right) \bar{V}_{L L}^{(s)}, \quad \tilde{V}_{S L}^{(s)} \equiv \bar{V}_{S L}^{(s)}, \quad$ and $\tilde{c}_{k, l}^{\left(n_{1}, n_{2}\right)}\left(\tilde{q}_{s}, \tilde{q}_{s^{\prime}} ; \tilde{t}\right) \equiv\left(\omega_{0} / \omega_{s}\right)^{n_{1}}\left(\omega_{0} / \omega_{s^{\prime}}\right)^{n_{2}} \bar{c}_{k, l}^{\left(n_{1}, n_{2}\right)}\left(\bar{q}_{s}, \bar{q}_{s^{\prime}} ; \bar{t}\right)$. The optical properties are also renormalized, with $\tilde{\mu}_{s}$ $\equiv\left(\omega_{0} / \omega_{s}\right) \bar{\mu}_{s}, \quad \tilde{\Pi}_{s} \equiv\left(\omega_{0} / \omega_{s}\right) \bar{\Pi}_{s}, \quad \tilde{\mu}_{s s^{\prime}} \equiv\left(\omega_{0}^{2} / \omega_{s} \omega_{s^{\prime}}\right) \bar{\mu}_{s s^{\prime}}$, and $\tilde{\Pi}_{s s^{\prime}} \equiv\left(\omega_{0}^{2} / \omega_{s} \omega_{s^{\prime}}\right) \bar{\Pi}_{s s^{\prime}}$, respectively. The values of these parameters that best reproduce the $1 \mathrm{D}$ and $2 \mathrm{D}$ vibrational spectra of liquid water are listed in Tables III and IV.

\section{APPENDIX C: 2D SPECTRAL ANALYSIS OF MODE-MODE INTERACTIONS USING MD AND BO MODEL APPROACHES}

In the 2D IR-Raman and 2D Raman spectra of liquid water obtained from MD simulations, the peaks representing the coupling between the HB-intermolecular vibrational and $\mathrm{OH}$-stretching modes were clearly observed, while those representing the coupling of other modes were unclear due to their weak peak intensities. To elucidate this fact, we decomposed the 2D spectra in terms of the permanent, induced, and mixed contributions of the dipole moment and polarizability elements. In this decomposition, the pure permanent and pure induced contributions in the 2D RII signal are, for example, expressed as $\left\langle\left\{\left\{\boldsymbol{\mu}^{P}\left(t_{2}\right), \boldsymbol{\mu}^{P}(0)\right\}_{\mathrm{PB}}, \boldsymbol{\Pi}^{P}\left(-t_{1}\right)\right\}_{\mathrm{PB}}\right\rangle$ and $\left\langle\left\{\left\{\boldsymbol{\mu}^{I}\left(t_{2}\right), \boldsymbol{\mu}^{I}(0)\right\}_{\mathrm{PB}}, \boldsymbol{\Pi}^{I}\left(-t_{1}\right)\right\}_{\mathrm{PB}}\right\rangle$, respectively, while the mixed contribution is expressed as the sum of six terms, one of which is expressed as $\left\langle\left\{\left\{\boldsymbol{\mu}^{I}\left(t_{2}\right), \boldsymbol{\mu}^{I}(0)\right\}_{\mathrm{PB}}, \boldsymbol{\Pi}^{P}\left(-t_{1}\right)\right\}_{\mathrm{PB}}\right\rangle$.

We present the 1D IR and 1D Raman spectra in terms of the total, permanent, induced, and mixed contributions obtained from the MD simulations in Fig. 8 and display the computational results for the $z z z z$ tensor element of (i) $2 \mathrm{D}$ RII, (ii) 2D IRI, and (iii) 2D IIR spectra and the $z z z z z z$ tensor element of (iv) 2D Raman spectra in terms of the (a) total, (b) permanent, (c) induced, and (d) their mixed contributions for liquid water obtained from the MD simulations in Fig. 8. Comparing the calculated total spectra presented in Fig. 9(a) with the decomposed spectra presented in Figs. 9(b)-9(d), we find that the main contribution to the $2 \mathrm{D}$ spectra arises from 
TABLE V. The parameter values of the dipole moment and polarizability for the LL+SL BO model fitted to reproduce the 1D and 2D spectra of the permanent contribution obtained from the MD simulations.

\begin{tabular}{lcclc}
\hline \hline$s$ & $\tilde{\mu}_{s}$ & $\tilde{\mu}_{s s}$ & $\tilde{\Pi}_{s}$ & $\tilde{\Pi}_{s s}$ \\
\hline 1 & 0.92 & $3.1 \times 10^{-3}$ & 1.5 & $6.2 \times 10^{-3}$ \\
2 & 1.6 & 0 & 0.23 & $-1.0 \times 10^{-2}$ \\
3 & 14.9 & 0 & 1.0 & -0.67 \\
4 & 8.7 & 0 & 0 & 0 \\
\hline$s-s^{\prime}$ & \multicolumn{5}{c}{$\tilde{\mu}_{s s^{\prime}}$} & & $\tilde{\Pi}_{s s^{\prime}}$ \\
\hline $1-2$ & 0 & & $1.3 \times 10^{-3}$ \\
$1-3$ & $5.1 \times 10^{-2}$ & & 0.10 \\
$1-4$ & $5.5 \times 10^{-2}$ & & 0 \\
$2-3$ & 0 & & 0 \\
$2-4$ & & $7.8 \times 10^{-3}$ & & 0 \\
$3-4$ & & $3.1 \times 10^{-2}$ & & \\
\hline \hline
\end{tabular}

the mixed contribution presented in Fig. 9(d). The labeled peaks in each spectrum correspond to the peaks with the same labels appearing in Fig. 5.

In all of the 2D spectra, we observe cross peaks between the $\mathrm{HB}$-intermolecular vibrational and $\mathrm{OH}$-stretching modes (labeled by "F") arising from the interaction, as discussed in Sec. IV, while we do not observe such cross peaks for the other modes, except for the permanent contributions presented in Fig. 9(b). For this reason, we employed the $\mathrm{LL}+\mathrm{SL} \mathrm{BO}$ model to fit the permanent contributions to the 1D and 2D spectra obtained from the MD simulations, as displayed in Figs. 10(a)-10(c). The parameter values for the optical properties are presented in Table $\mathrm{V}$, while those for the bath and the anharmonicity of the potential are presented in Tables III and IV. The 2D spectra for the (i) 2D RII, (ii) 2D IRI, (iii) 2D IIR, and (iv) 2D Raman cases of liquid water obtained from the MD simulations and the LL+SL BO model are presented in Figs. 10(b) and 10(c). On the basis of the BO analysis, we find that the spectral peaks labeled by "H" and "I" correspond to the cross peaks of the HB-intermolecular libration and $\mathrm{OH}$-stretching modes from the EAHC, which are expressed in terms of Eqs. (22) and (23) in the NL2 and NL3 cases.

The peaks near $\left(\omega_{1}, \omega_{2}\right)=(800,1600) \quad \mathrm{cm}^{-1}$ and $\left(\omega_{1}, \omega_{2}\right)=(1600,800) \mathrm{cm}^{-1}$ labeled by "J" in the 2D IRI spectrum can be attributed to the EAHC peak between the HB-intermolecular libration and $\mathrm{HOH}$-bending modes, while the overtone contribution of the HB-intermolecular librational mode labeled by "J" from NL2 is suppressed, because the nonlinearity of the permanent dipole moment is small. ${ }^{19}$ The peaks near $\left(\omega_{1}, \omega_{2}\right)=(800,1600) \mathrm{cm}^{-1}$ and $\left(\omega_{1}, \omega_{2}\right)=(1600,800) \mathrm{cm}^{-1}$ in the 2D IRI spectrum also become similar, because the overtone contribution is small.

We observe the cross peaks between the $\mathrm{OH}$-stretching and $\mathrm{HOH}$-bending modes from the MAHC at the points labeled by "D," " $\mathbf{D}$ '," and " $\mathbf{D}$ "." Because the nonlinear polarizability of the $\mathrm{HOH}$ bending mode is weak, the overtone contributions of the $\mathrm{HOH}$-bending mode described by the NL2 and NL3 processes that should appear at the points labeled by "D" and "D" are small.
${ }^{1}$ S. Mukamel, Principles of Nonlinear Optical Spectroscopy (Oxford University Press, Oxford, 1995).

${ }^{2}$ M. Cho, Two-Dimensional Optical Spectroscopy (CRC Press, 2009).

${ }^{3} \mathrm{P}$. Hamm and M. Zanni, Concepts and Methods of 2 D Infrared Spectroscopy (Cambridge University Press, 2011).

${ }^{4}$ S. Imoto, S. S. Xantheas, and S. Saito, J. Chem. Phys. 139, 044503 (2013).

${ }^{5}$ T. Yagasaki and S. Saito, Annu. Rev. Phys. Chem. 64, 55 (2013).

${ }^{6}$ S. Imoto, S. S. Xantheas, and S. Saito, J. Phys. Chem. B 119, 11068 (2015).

${ }^{7}$ J. Jeon and M. Cho, New J. Phys. 12, 065001 (2010).

${ }^{8}$ J. Jeon and M. Cho, J. Phys. Chem. B 118, 8148 (2014).

${ }^{9}$ T. Ishiyama, A. Morita, and T. Tahara, J. Chem. Phys. 142, 212407 (2015).

${ }^{10}$ Y. Nagata, Y. Tanimura, and S. Mukamel, J. Chem. Phys. 126, 204703 (2007).

${ }^{11}$ A. Ma and R. M. Stratt, Phys. Rev. Lett. 85, 1004 (2000).

${ }^{12}$ S. Saito and I. Ohmine, J. Chem. Phys. 119, 9073 (2003).

${ }^{13}$ S. Saito and I. Ohmine, J. Chem. Phys. 125, 084506 (2006).

${ }^{14}$ Y. Nagata and Y. Tanimura, J. Chem. Phys. 124, 024508 (2006).

${ }^{15}$ Y. Nagata, T. Hasegawa, and Y. Tanimura, J. Chem. Phys. 124, 194504 (2006).

${ }^{16}$ T. Hasegawa and Y. Tanimura, J. Chem. Phys. 125, 074512 (2006).

${ }^{17}$ H. Ito, J.-Y. Jo, and Y. Tanimura, Struct. Dyn. 2, 054102 (2015).

${ }^{18}$ H. Ito, T. Hasegawa, and Y. Tanimura, J. Chem. Phys. 141, 124503 (2014).

${ }^{19}$ T. Ikeda, H. Ito, and Y. Tanimura, J. Chem. Phys. 142, 212421 (2015).

${ }^{20}$ P. Hamm and J. Savolainen, J. Chem. Phys. 136, 094516 (2012).

${ }^{21}$ P. Hamm, J. Savolainen, J. Ono, and Y. Tanimura, J. Chem. Phys. 136, 236101 (2012).

${ }^{22}$ P. Hamm, J. Chem. Phys. 141, 184201 (2014).

${ }^{23}$ Z. Pan, T. Wu, T. Jin, Y. Liu, Y. Nagata, R. Zhang, and W. Zhuang, J. Chem. Phys. 142, 212419 (2015).

${ }^{24}$ Y. Tanimura and S. Mukamel, J. Chem. Phys. 99, 9496 (1993).

${ }^{25}$ K. Okumura and Y. Tanimura, Chem. Phys. Lett. 277, 159 (1997).

${ }^{26}$ K. Okumura, A. Tokmakoff, and Y. Tanimura, Chem. Phys. Lett. 314, 488 (1999).

${ }^{27}$ T. Steffen and Y. Tanimura, J. Phys. Soc. Jpn. 69, 3115 (2000).

${ }^{28}$ K. Okumura and Y. Tanimura, J. Chem. Phys. 106, 1687 (1997).

${ }^{29}$ K. Okumura and Y. Tanimura, J. Chem. Phys. 107, 2267 (1997).

${ }^{30}$ Y. Tanimura, Chem. Phys. 233, 217 (1998).

${ }^{31}$ K. Okumura and Y. Tanimura, Chem. Phys. Lett. 278, 175 (1997).

${ }^{32}$ A. Tokmakoff, M. J. Lang, D. S. Larsen, G. R. Fleming, V. Chernyak, and S. Mukamel, Phys. Rev. Lett. 79, 2702 (1997).

${ }^{33}$ M. Cho, K. Okumura, and Y. Tanimura, J. Chem. Phys. 108, 1326 (1998).

${ }^{34}$ K. Okumura, A. Tokmakoff, and Y. Tanimura, J. Chem. Phys. 111, 492 (1999).

${ }^{35}$ M. Cho, J. Chem. Phys. 111, 4140 (1999).

${ }^{36}$ M. L. Cowan, B. D. Bruner, N. Huse, J. R. Dwyer, B. Chugh, E. T. J. Nibbering, T. Elsaesser, and R. J. D. Miller, Nature (London) 434, 199 (2005).

${ }^{37}$ N. Huse, S. Ashihara, E. T. J. Nibbering, and T. Elsaesser, Chem. Phys. Lett. 404, 389 (2005).

${ }^{38}$ S. Ashihara, N. Huse, A. Espagne, E. T. J. Nibbering, and T. Elsaesser, Chem. Phys. Lett. 424, 66 (2006)

${ }^{39}$ A. Paarmann, T. Hayashi, S. Mukamel, and R. J. D. Miller, J. Chem. Phys. 128, 191103 (2008).

${ }^{40}$ T. L. C. Jansen, B. M. Auer, M. Yang, and J. L. Skinner, J. Chem. Phys. 132, 224503 (2010).

${ }^{41}$ C. J. Fecko, J. J. Loparo, S. T. Roberts, and A. Tokmakoff, J. Chem. Phys. 122, 054506 (2005).

${ }^{42}$ K. Ramasesha, L. D. Marco, A. Mandal, and A. Tokmakoff, Nat. Chem. 5, 935 (2013).

${ }^{43}$ M. Thamer, L. D. Marco, K. Ramasesha, A. Mandal, and A. Tokmakoff, Science 350, 78 (2015).

${ }^{44}$ M. Khalil, N. Demirdoven, and A. Tokmakoff, J. Phys. Chem. A 107, 5258 (2003).

${ }^{45}$ J. Savolainen, S. Ahmed, and P. Hamm, Proc. Natl. Acad. Sci. U. S. A. 110, 20402 (2013).

${ }^{46}$ T. Hasegawa and Y. Tanimura, J. Phys. Chem. B 115, 5545 (2011).

${ }^{47}$ K. Okumura and Y. Tanimura, Phys. Rev. E 56, 2747 (1997).

${ }^{48}$ A. Ishizaki and Y. Tanimura, J. Chem. Phys. 125, 084501 (2006).

${ }^{49}$ A. Sakurai and Y. Tanimura, J. Phys. Chem. A 115, 4009 (2011).

${ }^{50}$ A. Ishizaki and Y. Tanimura, J. Phys. Chem. A 111, 9269 (2007).

${ }^{51}$ Y. Tanimura and A. Ishizaki, Acc. Chem. Res. 42, 1270 (2009).

${ }^{52}$ Y. Tanimura and R. Kubo, J. Phys. Soc. Jpn. 58, 101 (1989).

${ }^{53}$ Y. Tanimura, J. Chem. Phys. 141, 044114 (2014).

${ }^{54}$ Y. Tanimura and P. G. Wolynes, Phys. Rev. A 43, 4131 (1991). 
${ }^{55}$ Y. Tanimura and P. G. Wolynes, J. Chem. Phys. 96, 8485 (1992).

${ }^{56}$ Y. Tanimura, J. Chem. Phys. 142, 144110 (2015).

${ }^{57}$ Y. Tanimura, J. Phys. Soc. Jpn. 75, 082001 (2006).

${ }^{58}$ Y. Tanimura and T. Steffen, J. Phys. Soc. Jpn. 69, 4095 (2000).

${ }^{59}$ T. Kato and Y. Tanimura, J. Chem. Phys. 117, 6221 (2002).

${ }^{60}$ T. Kato and Y. Tanimura, J. Chem. Phys. 120, 260 (2004).

${ }^{61}$ S. Saito and I. Ohmine, J. Chem. Phys. 108, 240 (1998).

${ }^{62}$ P. H. Berens and K. R. Wilson, J. Chem. Phys. 74, 4872 (1981).

${ }^{63}$ A. J. Stone and M. Alderton, Mol. Phys. 56, 1047 (1985).

${ }^{64}$ See supplementary material at http://dx.doi.org/10.1063/1.4941842 for the details of the full-order CFDID polarizability model and the POLI2VS potential mode.
${ }^{65}$ J. Sala, E. Guárdia, and M. Masia, J. Chem. Phys. 133, 234101 (2010).

${ }^{66}$ H. Frostig, T. Bayer, N. Dudovich, Y. C. Eldar, and Y. Silberberg, Nat. Photonics 9, 339 (2015).

${ }^{67}$ T. Yagasaki and S. Saito, J. Chem. Phys. 128, 154521 (2008).

${ }^{68}$ Y. Nagata, S. Yoshimune, C.-S. Hsieh, J. Hunger, and M. Bonn, Phys. Rev. X 5, 021002 (2015).

${ }^{69}$ J. Liu, W. H. Miller, G. S. Fanourgakis, S. S. Xantheas, S. Imoto, and S. Saito, J. Chem. Phys. 135, 244503 (2011).

${ }^{70}$ J. Liu, Int. J. Quantum Chem. 115, 657 (2015).

${ }^{71}$ G. R. Medders and F. Paesani, J. Chem. Theory Comput. 11, 1145 (2015).

${ }^{72}$ H. Risken, The Fokker-Planck Equation, 2nd ed. (Springer, Berlin, 1989). 